\title{
The impact of dual bronchodilation on cardiovascular serious adverse events and mortality in COPD: a quantitative synthesis
}

This article was published in the following Dove Press journal: International Journal of COPD

\author{
Paola Rogliani ${ }^{1,2}$ \\ Maria Gabriella Matera ${ }^{3}$ \\ Josuel Ora \\ Mario Cazzola' \\ Luigino Calzetta' \\ 'Department of Experimental \\ Medicine and Surgery, University \\ of Rome Tor Vergata, Rome, Italy; \\ ${ }^{2}$ Division of Respiratory Medicine, \\ University Hospital Tor Vergata, \\ Rome, Italy; ${ }^{3}$ Department of \\ Experimental Medicine, University of \\ Campania Luigi Vanvitelli, Naples, Italy
}

Correspondence: Paola Rogliani Department of Experimental Medicine and Surgery, University of Rome Tor Vergata, Via Montpellier I, 00133 , Roma, Italy Email paola.rogliani@uniroma2.it
Objective: Long-acting $\beta_{2}$-agonists (LABAs) and long-acting muscarinic antagonists (LAMAs) are burdened by the potential risk of inducing cardiovascular serious adverse events (SAEs) in COPD patients. Since the risk of combining a LABA with a LAMA could be greater, we have carried out a quantitative synthesis to investigate the cardiovascular safety profile of LABA/LAMA fixed-dose combinations (FDCs).

Methods: A pair-wise and network meta-analysis was performed by using the data of the repository database ClinicalTrials. gov concerning the impact of approved LABA/LAMA FDCs versus monocomponents and/or placebo on cardiovascular SAEs in COPD.

Results: Overall, LABA/LAMA FDCs did not significantly $(P>0.05)$ modulate the risk of cardiovascular SAEs versus monocomponents. However, the network meta-analysis indicated that aclidinium/formoterol $400 / 12 \mu \mathrm{g}$ and tiotropium/olodaterol $5 / 5 \mu \mathrm{g}$ were the safest FDCs, followed by umeclidinium/vilanterol $62.5 / 25 \mu \mathrm{g}$ which was as safe as placebo, whereas glycopyrronium/formoterol 14.9/9.6, glycopyrronium/indacaterol 15.6/27.5 $\mu \mathrm{g}$, and glycopyrronium/ indacaterol 50/110 $\mu \mathrm{g}$ were the least safe FDCs. No impact on mortality was detected for each specific FDC.

Conclusion: This meta-analysis indicates that LABA/LAMA FDC therapy is characterized by an excellent cardiovascular safety profile in COPD patients. However, the findings of this quantitative synthesis have been obtained from populations that participated in randomized clinical trials, and were devoid of major cardiovascular diseases. Thus, post-marketing surveillance and observational studies may help to better define the real impact of specific FDCs with regard to the cardiovascular risk.

Keywords: COPD, LABA/LAMA FDC, cardiovascular safety, mortality, meta-analysis

\section{Introduction}

Dual bronchodilation therapy is the cornerstone for the treatment of COPD, and a large body of evidence indicates that combining a long-acting $\beta_{2}$-agonist (LABA) with a long-acting muscarinic antagonist (LAMA) leads to synergistic bronchorelaxant effect. ${ }^{1-5}$ Although some fixed-dose combinations (FDCs) elicit prevalently additive interaction when administered at the concentration-ratio currently available in the market, ${ }^{6,7}$ the beneficial interaction between LABAs and LAMAs is a pharmacological characteristic that would allow reduction of the doses of each monocomponent in order to optimize bronchodilation and, thus, reduce the risk of adverse events (AEs). ${ }^{8}$

Unfortunately, the doses of each single bronchodilator included in the currently available LABA/LAMA FDCs have not been modified with respect to the doses of medications containing single-agents. Such an approach may appear simplistic, as it 
does not allow modulation of the doses of the dual bronchodilation therapy accordingly with the characteristics of COPD patients, namely clinical conditions and airflow limitation. ${ }^{9}$ Furthermore, it can raise concerns with regard to the safety profile of LABA/LAMA FDCs. In fact, since both LABAs and LAMAs administered as monocomponents at the full doses approved for the treatment of COPD are burdened by the potential risk of inducing cardiovascular serious AEs (SAEs), the risk of combining an LABA with an LAMA could be even greater. ${ }^{10}$

Results of a recent meta-analysis did not show any significant difference concerning the cardiac safety profile of LABA/ LAMA FDCs compared with their monocomponents. ${ }^{11}$ Nevertheless, several studies were not included in that previous analysis because no suitable data on cardiac SAEs were reported, ${ }^{11}$ no randomized clinical trials (RCTs) were available for all the currently approved FDCs, and the vascular safety profile was not investigated.

Since the question about the real impact of LABA/LAMA FDCs on the cardiovascular system is still open, we have carried out a pair-wise meta-analysis in order to characterize the cardiovascular safety profile and mortality of each
LABA/LAMA FDC currently approved for the treatment of COPD. Furthermore, since a well-performed quantitative synthesis allows for indirect comparisons of multiple interventions that have not been studied in a head-to-head fashion, ${ }^{12}$ we have also carried out a network meta-analysis in order to compare the cardiovascular safety profile of the approved LABA/LAMA FDCs.

\section{Methods}

\section{Search strategy}

This pair-wise and network meta-analysis has been registered in PROSPERO (registration number: CRD42017070100; available from: https://www.crd.york.ac.uk/PROSPERO/ display record.asp?ID=CRD42017070100), and performed in agreement with the Preferred Reporting Items for Systematic Reviews and Meta-Analyses (PRISMA) Statement (Figure 1). ${ }^{13}$ Furthermore, this synthesis satisfied all the recommended items reported by the PRISMA-P 2015 checklist. ${ }^{14}$

We undertook a comprehensive literature search for RCTs evaluating the impact of dual bronchodilation on the risk of cardiovascular SAEs in patients suffering from COPD, diagnosed by pulmonary function testing.

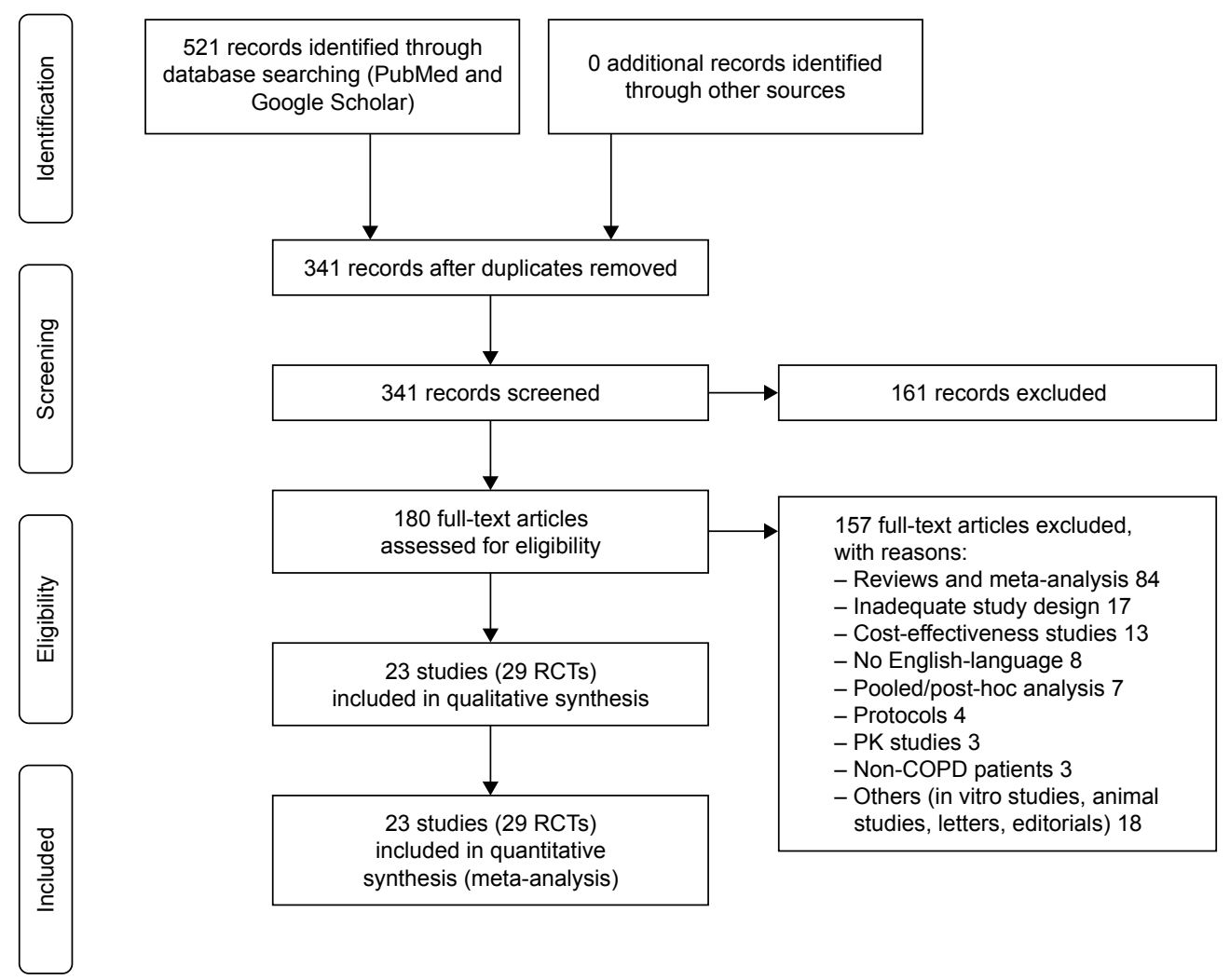

Figure I PRISMA flow diagram for the identification of studies included in the meta-analysis concerning the impact of LABA/LAMA FDCs on cardiovascular SAEs in COPD patients.

Abbreviations: COPD, chronic obstructive pulmonary disease; FDCs, fixed-dose combinations; LABAs, long-acting $\beta_{2}$-agonists; LAMAs, long-acting muscarinic antagonists; PK, pharmacokinetic; RCT, randomized controlled trial; SAEs, serious adverse events. 
The LABA/LAMA FDCs currently approved in COPD by the European Medicines Agency and/or US Food and Drug Administration were searched. In particular, aclidinium/ formoterol 400/12 $\mu \mathrm{g}$ (A/F 400/12), glycopyrronium/ indacaterol 15.6/27.5 $\mu \mathrm{g}$ (G/I 15.6/27.5), glycopyrronium/ indacaterol 50/110 $\mu \mathrm{g}(\mathrm{G} / \mathrm{I} 50 / 110)$, umeclidinium/vilanterol 62.5/25 $\mu \mathrm{g}$ (U/V 62.5/25), tiotropium/olodaterol 5/5 $\mu \mathrm{g}$ (T/O 5/5), and glycopyrronium/formoterol 14.9/9.6 $\mu \mathrm{g}$ (G/F 14.4/9.6) were searched for the FDCs, and the terms "chronic obstructive pulmonary disease" and/or "COPD" were searched for the disease. The search was performed in PubMed, Scopus, Embase, Google Scholar and the repository database ClinicalTrials.gov through June 2017, ${ }^{15}$ in order to identify relevant studies reported in English and published up to June 31, 2017. Citations of previously published metaanalyses and relevant reviews were checked to select further pertinent studies, if any. ${ }^{10,11,16-18}$

Two reviewers independently checked the relevant RCTs identified from literature searches and databases. RCTs were selected in agreement with the previously mentioned criteria, and any difference in opinion about eligibility was resolved by consensus.

\section{Study selection}

RCTs reporting in the repository database ClinicalTrials.gov raw data concerning the impact of the approved LABA/ LAMA FDCs versus monocomponents and/or placebo on cardiovascular SAEs in COPD patients were selected, and those reporting at least one cardiovascular SAE were included in the meta-analysis. No restriction on the duration of the treatment was applied. Two reviewers independently examined the clinical trials and any difference in opinion about eligibility was resolved by consensus.

\section{Data extraction}

Data from included studies were extracted from published papers, and/or online supplementary files, and/or the public database ClinicalTrials.gov. Data extraction was carried out in agreement with the recommendations provided by the Cochrane Handbook for Systematic Reviews of Interventions. ${ }^{19}$ Data were extracted and checked for study characteristics and duration, doses of medications, patient characteristics, age, gender, smoking habits, forced expiratory volume in 1 second $\left(\mathrm{FEV}_{1}\right)$, cardiovascular SAEs, and Jadad score.

\section{Endpoints}

The primary endpoint of this quantitative synthesis was to assess the cardiovascular safety profile (SAEs) of
LABA/LAMA FDCs administered at the currently approved doses in COPD patients, compared with the monocomponents included in the FDCs.

The secondary endpoints were, 1) the influence of the currently approved LABA/LAMA FDCs on mortality in COPD patients, compared with the monocomponents included in the FDCs, and 2) the indirect safety comparison on cardiovascular SAEs among the currently approved LABA/LAMA FDCs compared with placebo.

\section{Quality score, risk of bias and evidence profile}

The Jadad score, with a scale of $1-5$ (score of 5 being the best quality), was used to assess the quality of the RCTs concerning the likelihood of biases related to randomization, double-blinding, withdrawals and dropouts. ${ }^{11}$ Two reviewers independently assessed the quality of individual studies, and any difference in opinion about the quality score was resolved by consensus.

The risk of publication bias was assessed by applying the funnel plot and Egger's test through the following regression equation: $\mathrm{SND}=\mathrm{a}+\mathrm{b} \times$ precision, where $\mathrm{SND}$ represents the standard normal deviation (treatment effect divided by its standard error $[\mathrm{SE}]$ ), and precision represents the reciprocal of the SE. Evidence of asymmetry from Egger's test was considered to be significant at $P<0.1$, and the graphical representation of $90 \%$ confidence bands are presented. ${ }^{11}$

The optimal information size (OIS) was calculated as previously described ${ }^{20}$ and the quality of the evidence has been assessed in agreement with the Grading of Recommendations Assessment, Development, and Evaluation (GRADE) system. ${ }^{21}$

\section{Data analysis}

We performed both a pair-wise and network meta-analysis to evaluate the cardiovascular safety profile of LABA/LAMA FDCs in COPD patients. Since the follow-up duration was not consistent among the RCTs included in this meta-analysis, the data have been normalized as a function of person-year. ${ }^{22-24}$ This method involved the conversion of the measures into a common metric (events per person-time) prior to metaanalysis of the data, leading to increased estimates of effect, precision, and clinical interpretability of results. ${ }^{19,25}$

Results are expressed as Risk Ratio (RR) and 95\% Cl in pair-wise meta-analysis. Since data were selected from a series of studies performed by researchers operating independently, and a common effect size cannot be assumed, we used the random-effects model to perform the pair-wise 
meta-analysis in order to balance the study weights and adequately estimate the $95 \%$ CI of the mean distribution of drugs effect on the investigated variable. ${ }^{22}$ In fact, although the mathematics behind the fixed-effects model are much simpler than those of the random-effects model, results of this quantitative synthesis cannot be generalized via fixed-effects model since the included studies were quite dissimilar, ${ }^{26}$ as reported in Table 1 . Therefore, the greater the degree of difference among the studies incorporated in the analysis, the more important it becomes to employ the random-effects model. ${ }^{27}$

Subset analyses were performed with regard to the effect of the class of monocomponents included in the FDCs (LABAs or LAMAs) and each specific FDC. High quality studies were identified as having Jadad score $\geq 3 .{ }^{28}$

The network meta-analysis was performed to indirectly compare the effect of specific FDCs. A full Bayesian evidence network was used (chains: 4; initial values scaling: 2.5 ; tuning

Table I Patient demographics, baseline and study characteristics

\begin{tabular}{|c|c|c|c|c|c|c|}
\hline $\begin{array}{l}\text { Study and } \\
\text { year }\end{array}$ & $\begin{array}{l}\text { ClinicalTrials. } \\
\text { gov identifier }\end{array}$ & Study characteristics & $\begin{array}{l}\text { Duration } \\
\text { of study } \\
\text { (weeks) }\end{array}$ & $\begin{array}{l}\text { Number of } \\
\text { analyzed } \\
\text { patients }\end{array}$ & Drugs (doses) & $\begin{array}{l}\text { Inhaler device } \\
\text { (brand) }\end{array}$ \\
\hline $\begin{array}{l}\text { D’Urzo et al } \\
(2017)^{36}\end{array}$ & NCT0I 572792 & $\begin{array}{l}\text { Randomized, double-blind, } \\
\text { parallel-group, placebo- } \\
\text { and active-controlled }\end{array}$ & 28 & 714 & $\begin{array}{l}\text { Aclidinium/ } \\
\text { formoterol } \\
400 / 12 \mu \mathrm{g}\end{array}$ & $\begin{array}{l}\text { Dry powder inhaler } \\
\left(\text { Genuair }^{\circledR} / \text { Pressair }^{\circledR}\right)\end{array}$ \\
\hline $\begin{array}{l}\text { Donohue } \\
\text { et al }(2016)^{37}\end{array}$ & NCT0I 437540 & $\begin{array}{l}\text { Randomized, double-blind, } \\
\text { parallel-group, active- } \\
\text { controlled }\end{array}$ & 52 & 590 & $\begin{array}{l}\text { Aclidinium/ } \\
\text { formoterol } \\
400 / 12 \mu \mathrm{g}\end{array}$ & $\begin{array}{l}\text { Dry powder inhaler } \\
\text { (Genuair/Pressair) }\end{array}$ \\
\hline $\begin{array}{l}\text { Singh et al } \\
(2014)^{38}\end{array}$ & NCT0I462942 & $\begin{array}{l}\text { Multicenter, randomized, } \\
\text { double-blind, parallel- } \\
\text { group, active- and placebo- } \\
\text { controlled }\end{array}$ & 24 & $\mathrm{I}, 348$ & $\begin{array}{l}\text { Aclidinium/ } \\
\text { formoterol } \\
400 / 12 \mu g\end{array}$ & $\begin{array}{l}\text { Dry powder inhaler } \\
\text { (Genuair/Pressair) }\end{array}$ \\
\hline $\begin{array}{l}\text { D’Urzo et al } \\
(2014)^{39}\end{array}$ & NCT0I437397 & $\begin{array}{l}\text { Multicentre, randomized, } \\
\text { double-blind, placebo- } \\
\text { controlled }\end{array}$ & 24 & 1,389 & $\begin{array}{l}\text { Aclidinium/ } \\
\text { formoterol } \\
400 / 12 \mu g\end{array}$ & $\begin{array}{l}\text { Dry powder inhaler } \\
\text { (Genuair/Pressair) }\end{array}$ \\
\hline $\begin{array}{l}\text { Mahler et al } \\
(2015)^{40}\end{array}$ & $\begin{array}{l}\text { NCT0I727I4I, } \\
\text { NCT0I7I25I6 }\end{array}$ & $\begin{array}{l}\text { Identical, multicenter, } \\
\text { randomized, double-blind, } \\
\text { parallel-group, placebo- } \\
\text { and active-controlled }\end{array}$ & 12 & 2,040 & $\begin{array}{l}\text { Glycopyrronium/ } \\
\text { indacaterol } \\
\text { 15.6/27.5 } \mu \mathrm{g}\end{array}$ & $\begin{array}{l}\text { Dry powder inhaler } \\
\left(\text { Neohaler }^{\circledR}\right)\end{array}$ \\
\hline $\begin{array}{l}\text { Watz et al } \\
(2016)^{4 !}\end{array}$ & NCT01996319 & $\begin{array}{l}\text { Multicenter, randomized, } \\
\text { double-blind, placebo- } \\
\text { controlled, crossover }\end{array}$ & 3 & 193 & $\begin{array}{l}\text { Glycopyrronium/ } \\
\text { indacaterol } \\
50 / 110 \mu \mathrm{g}\end{array}$ & NA \\
\hline $\begin{array}{l}\text { Buhl et al } \\
(2015)^{44}\end{array}$ & NCT0II207I7 & $\begin{array}{l}\text { Multicenter, randomized, } \\
\text { double-blind, placebo- } \\
\text { controlled, parallel-group, } \\
\text { dummy }\end{array}$ & 52 & 338 & $\begin{array}{l}\text { Glycopyrronium/ } \\
\text { indacaterol } \\
50 / 110 \mu \mathrm{g}\end{array}$ & $\begin{array}{l}\text { Dry powder inhaler } \\
\text { (NA) }\end{array}$ \\
\hline $\begin{array}{l}\text { Bateman et al } \\
(2013)^{42}\end{array}$ & NCT0I 202188 & $\begin{array}{l}\text { Multicenter, randomized, } \\
\text { double-blind, parallel- } \\
\text { group, placebo- and active- } \\
\text { controlled }\end{array}$ & 26 & 1,179 & $\begin{array}{l}\text { Glycopyrronium/ } \\
\text { indacaterol } \\
50 / 110 \mu g\end{array}$ & $\begin{array}{l}\text { Dry powder inhaler } \\
\left(\text { Breezhaler }^{\circledR}\right)\end{array}$ \\
\hline $\begin{array}{l}\text { Wedzicha } \\
\text { et al }(2013)^{43}\end{array}$ & NCT0II 2069I & $\begin{array}{l}\text { Multicenter, randomized, } \\
\text { double-blind, parallel-group }\end{array}$ & 64 & 1,469 & $\begin{array}{l}\text { Glycopyrronium/ } \\
\text { indacaterol } \\
50 / 110 \mu \mathrm{g}\end{array}$ & $\begin{array}{l}\text { Dry powder inhaler } \\
\text { (Breezhaler) }\end{array}$ \\
\hline $\begin{array}{l}\text { O’Donnell } \\
\text { et al }(20 \mid 7)^{45}\end{array}$ & $\begin{array}{l}\text { NCTOI533922, } \\
\text { NCT0I533935 }\end{array}$ & $\begin{array}{l}\text { Replicate, randomized, } \\
\text { double-blind, placebo- } \\
\text { controlled, incomplete- } \\
\text { crossover }\end{array}$ & 6 & 450 & $\begin{array}{l}\text { Tiotropium/ } \\
\text { olodaterol 5/5 } \mu g\end{array}$ & $\begin{array}{l}\text { Soft mist inhaler } \\
\left.\text { (Respimat }^{\circledR}\right)\end{array}$ \\
\hline
\end{tabular}


iterations: 20,000; simulation iterations: 50,000; tuning interval: 10), and the convergence diagnostics for consistency and inconsistency were assessed via the Brooks-Gelman-Rubin method, as previously reported. ${ }^{29}$ Due to the characteristics of parameters besides the available data, the just proper non-informative distributions specified the prior densities, in agreement with the Bayesian Approaches to Clinical Trials and Health-Care Evaluation. ${ }^{30,31}$ Since the distributions were sufficiently vague, the reference treatment, study baseline effects, and heterogeneity variance were unlikely to have a noticeable impact on model results. In this condition, GeMTC software automatically generates and runs the required Bayesian hierarchical model and selects the prior distributions and starting values as well, via heuristically determining a value for the outcome scale parameter (ie, outcome scale S). ${ }^{32,33}$ The posterior mean deviance of data points in the unrelated mean effects model was plotted against the posterior mean deviance in the consistency model in order to provide information for

\begin{tabular}{|c|c|c|c|c|c|c|c|}
\hline $\begin{array}{l}\text { Administration } \\
\text { regimen }\end{array}$ & Patient characteristics & $\begin{array}{l}\text { Age } \\
\text { (years) }\end{array}$ & $\begin{array}{l}\text { Male } \\
(\%)\end{array}$ & $\begin{array}{l}\text { Current } \\
\text { smokers } \\
\text { (\%) }\end{array}$ & $\begin{array}{l}\text { Smoking } \\
\text { history } \\
\text { (pack- } \\
\text { years) }\end{array}$ & $\begin{array}{l}\text { Post- } \\
\text { bronchodilator } \\
\text { FEV } \\
\text { (\% predicted) }\end{array}$ & $\begin{array}{l}\text { Jadad } \\
\text { score }\end{array}$ \\
\hline Twice daily & $\begin{array}{l}\text { Moderate-to-severe COPD } \\
\text { (post-bronchodilator } \mathrm{FEV}_{\mathrm{I}} / \\
\mathrm{FVC}<0.7 ; \mathrm{FEV}, \geq 30 \% \\
\text { and }<80 \% \text { predicted) }\end{array}$ & 63.2 & 51.1 & 54.9 & 53.3 & 53.3 & 5 \\
\hline Twice daily & $\begin{array}{l}\text { Moderate-to-severe COPD } \\
\text { (post-bronchodilator } \mathrm{FEV}_{\mathrm{I}} / \\
\mathrm{FVC}<0.7 ; \mathrm{FEV}, \geq 30 \% \\
\text { and }<80 \% \text { predicted) }\end{array}$ & 64.3 & 55.1 & 45.4 & 51.8 & 51.2 & 4 \\
\hline Twice daily & $\begin{array}{l}\text { Moderate-to-severe COPD } \\
\text { (post-bronchodilator } \mathrm{FEV}_{\mathrm{I}} / \\
\mathrm{FVC}<0.7 ; \mathrm{FEV}, \geq 30 \% \\
\text { and }<80 \% \text { predicted) }\end{array}$ & 63.2 & 67.6 & 47.3 & $>10$ & 54.3 & 4 \\
\hline Twice daily & $\begin{array}{l}\text { Moderate-to-severe stable } \\
\text { COPD (post-bronchodilator } \\
\mathrm{FEV}_{\mathrm{l}} / \mathrm{FVC}<0.7 ; \mathrm{FEV}_{\mathrm{l}} \geq 30 \% \\
\text { and }<80 \% \text { predicted) }\end{array}$ & 64.1 & 53.3 & 51.7 & 52.5 & 53.7 & 3 \\
\hline Twice daily & $\begin{array}{l}\text { Stable COPD (post- } \\
\text { bronchodilator } \mathrm{FEV}_{\text {, }} / \mathrm{FVC} \\
<0.7 ; \mathrm{FEV}, \geq 30 \% \text { and } \\
<80 \% \text { predicted) }\end{array}$ & 63.5 & 64.8 & 52.2 & $>10$ & 54.6 & 5 \\
\hline Once daily & $\begin{array}{l}\text { Moderate-to-severe stable } \\
\text { COPD (post-bronchodilator } \\
\text { FEV,/FVC <0.7; FEV, } \\
40 \%-80 \% \text { predicted) }\end{array}$ & 62.8 & 65.5 & 56.7 & 47.5 & 61.6 & 4 \\
\hline Once daily & $\begin{array}{l}\text { Moderate-to-severe COPD } \\
\text { (post-bronchodilator } \mathrm{FEV}_{\mathrm{I}} / \\
\mathrm{FVC}<0.7 ; \mathrm{FEV}, \geq 30 \% \\
\text { and }<80 \% \text { predicted) }\end{array}$ & 62.7 & 76.9 & 49.2 & $4 I . I$ & 53.3 & 5 \\
\hline Once daily & $\begin{array}{l}\text { Moderate-to-severe stable } \\
\text { COPD (post-bronchodilator } \\
\mathrm{FEV}_{\mathrm{I}} / \mathrm{FVC}<0.7 ; \mathrm{FEV}_{\mathrm{l}} \geq 30 \% \\
\text { and }<80 \% \text { predicted) }\end{array}$ & 64.0 & 77.2 & 40.0 & $>10$ & 55.2 & 4 \\
\hline Once daily & $\begin{array}{l}\text { Severe-to-very severe } \\
\text { COPD (post-bronchodilator } \\
\text { FEV }_{\text {l }} \text { FVC }<0.7 ; \text { FEV }_{1}<50 \% \\
\text { predicted) }\end{array}$ & 63.3 & 75.0 & 36.6 & $>10$ & 37.2 & 5 \\
\hline Once daily & $\begin{array}{l}\text { COPD (post-bronchodilator } \\
\mathrm{FEV}_{\mathrm{l}} / \mathrm{FVC}<0.7 ; \mathrm{FEV}_{1} \geq 30 \% \\
\text { and }<80 \% \text { predicted) }\end{array}$ & 61.7 & 71.2 & 39.1 & 45.8 & 52.0 & 3 \\
\hline
\end{tabular}


Table I (Continued)

\begin{tabular}{|c|c|c|c|c|c|c|}
\hline $\begin{array}{l}\text { Study and } \\
\text { year }\end{array}$ & $\begin{array}{l}\text { ClinicalTrials. } \\
\text { gov identifier }\end{array}$ & Study characteristics & $\begin{array}{l}\text { Duration } \\
\text { of study } \\
\text { (weeks) }\end{array}$ & $\begin{array}{l}\text { Number of } \\
\text { analyzed } \\
\text { patients }\end{array}$ & Drugs (doses) & $\begin{array}{l}\text { Inhaler device } \\
\text { (brand) }\end{array}$ \\
\hline $\begin{array}{l}\text { Ichinose et al } \\
(2017)^{46}\end{array}$ & NCT0I536262 & $\begin{array}{l}\text { Multicenter, randomized, } \\
\text { double-blind, parallel-group }\end{array}$ & 52 & 82 & $\begin{array}{l}\text { Tiotropium/ } \\
\text { olodaterol 5/5 } \mu \mathrm{g}\end{array}$ & $\begin{array}{l}\text { Soft mist inhaler } \\
\text { (Respimat) }\end{array}$ \\
\hline $\begin{array}{l}\text { Troosters } \\
\text { et al }(2016)^{47}\end{array}$ & NCT02085I6I & $\begin{array}{l}\text { Randomized, partially } \\
\text { double-blind, placebo- } \\
\text { controlled, parallel-group }\end{array}$ & 12 & 227 & $\begin{array}{l}\text { Tiotropium/ } \\
\text { olodaterol } 5 / 5 \mu \mathrm{g}\end{array}$ & $\begin{array}{l}\text { Soft mist inhaler } \\
\text { (Respimat) }\end{array}$ \\
\hline $\begin{array}{l}\text { Beeh et al } \\
(2015)^{48}\end{array}$ & NCT0I559II6 & $\begin{array}{l}\text { Multicenter randomized, } \\
\text { double-blind, placebo- } \\
\text { controlled, incomplete- } \\
\text { crossover }\end{array}$ & 6 & 139 & $\begin{array}{l}\text { Tiotropium/ } \\
\text { olodaterol } 5 / 5 \mu g\end{array}$ & $\begin{array}{l}\text { Soft mist inhaler } \\
\text { (Respimat) }\end{array}$ \\
\hline $\begin{array}{l}\text { Buhl et al } \\
(2015)^{49}\end{array}$ & $\begin{array}{l}\text { NCT0I43I } 274 \\
\text { NCT0I } 431287\end{array}$ & $\begin{array}{l}\text { Multicenter, multinational, } \\
\text { replicate, randomized, } \\
\text { double-blind, parallel- } \\
\text { group, active-controlled, } \\
\text { five-arm }\end{array}$ & 52 & 3,100 & $\begin{array}{l}\text { Tiotropium/ } \\
\text { olodaterol 5/5 } \mu \mathrm{g}\end{array}$ & $\begin{array}{l}\text { Soft mist inhaler } \\
\text { (Respimat) }\end{array}$ \\
\hline $\begin{array}{l}\text { Singh et al } \\
(2015)^{80}\end{array}$ & $\begin{array}{l}\text { NCT01964352, } \\
\text { NCT02006732 }\end{array}$ & $\begin{array}{l}\text { Multinational, replicate, } \\
\text { randomized, double-blind, } \\
\text { placebo-controlled, parallel } \\
\text { group }\end{array}$ & 12 & 1,217 & $\begin{array}{l}\text { Tiotropium/ } \\
\text { olodaterol 5/5 } \mu \mathrm{g}\end{array}$ & $\begin{array}{l}\text { Soft mist inhaler } \\
\text { (Respimat) }\end{array}$ \\
\hline $\begin{array}{l}\text { Donohue } \\
\text { et al }(2013)^{51}\end{array}$ & NCT0I3I3650 & $\begin{array}{l}\text { Multicenter, randomized, } \\
\text { double-blind, parallel- } \\
\text { group, placebo-controlled }\end{array}$ & 24 & 1,532 & $\begin{array}{l}\text { Umeclidinium/ } \\
\text { vilanterol } \\
62.5 / 25 \mu g\end{array}$ & $\begin{array}{l}\text { Dry powder inhaler } \\
\text { (NA) }\end{array}$ \\
\hline $\begin{array}{l}\text { Decramer } \\
\text { et al }(2014)^{52}\end{array}$ & NCT0I316900 & $\begin{array}{l}\text { Multicenter, randomized, } \\
\text { double-blind, parallel- } \\
\text { group, double-dummy }\end{array}$ & 24 & 421 & $\begin{array}{l}\text { Umeclidinium/ } \\
\text { vilanterol } \\
62.5 / 25 \mu g\end{array}$ & $\begin{array}{l}\text { Dry powder inhaler } \\
\left(\text { Ellipta }^{\circledR}\right)\end{array}$ \\
\hline $\begin{array}{l}\text { Siler et al } \\
(2016)^{53}\end{array}$ & NCT02I 52605 & $\begin{array}{l}\text { Multicenter, randomized, } \\
\text { double-blind, placebo- } \\
\text { controlled, parallel-group }\end{array}$ & 12 & 496 & $\begin{array}{l}\text { Umeclidinium/ } \\
\text { vilanterol } \\
62.5 / 25 \mu g\end{array}$ & $\begin{array}{l}\text { Dry powder inhaler } \\
\text { (Ellipta) }\end{array}$ \\
\hline $\begin{array}{l}\text { Donohue } \\
\text { et al }(2016)^{6}\end{array}$ & NCT0I7I6520 & $\begin{array}{l}\text { Multicenter, randomized, } \\
\text { double-blind, three-way, } \\
\text { complete block cross-over }\end{array}$ & 2 & 173 & $\begin{array}{l}\text { Umeclidinium/ } \\
\text { vilanterol } \\
62.5 / 25 \mu g\end{array}$ & $\begin{array}{l}\text { Dry powder inhaler } \\
\text { (Ellipta) }\end{array}$ \\
\hline $\begin{array}{l}\text { Zheng et al } \\
(2015)^{54}\end{array}$ & NCT0I6367I3 & $\begin{array}{l}\text { Multicenter, randomized, } \\
\text { double-blind, placebo- } \\
\text { controlled, parallel-group }\end{array}$ & 24 & 387 & $\begin{array}{l}\text { Umeclidinium/ } \\
\text { vilanterol } \\
62.5 / 25 \mu \mathrm{g}\end{array}$ & $\begin{array}{l}\text { Dry powder inhaler } \\
\text { (NA) }\end{array}$ \\
\hline $\begin{array}{l}\text { Maltais et al } \\
(20 \mid 4)^{55}\end{array}$ & $\begin{array}{l}\text { NCT0I } 323660 \text {, } \\
\text { NCT0I } 328444\end{array}$ & $\begin{array}{l}\text { Multicenter, randomized, } \\
\text { placebo-controlled, } \\
\text { parallel-group }\end{array}$ & 12 & 832 & $\begin{array}{l}\text { Umeclidinium/ } \\
\text { vilanterol } \\
62.5 / 25 \mu \mathrm{g}\end{array}$ & $\begin{array}{l}\text { Dry powder inhaler } \\
\text { (Ellipta) }\end{array}$ \\
\hline $\begin{array}{l}\text { Martinez } \\
\text { et al }(20 \mid 7)^{56}\end{array}$ & $\begin{array}{l}\text { NCT0I } 854645 \text {, } \\
\text { NCT0I } 854658\end{array}$ & $\begin{array}{l}\text { Multicenter, randomized, } \\
\text { double-blind, placebo- } \\
\text { controlled, parallel-group }\end{array}$ & 24 & 3,259 & $\begin{array}{l}\text { Glycopyrronium } \% \\
\text { formoterol } \\
\text { 14.4/9.6 } \mu \mathrm{g}\end{array}$ & $\begin{array}{l}\text { Pressurised } \\
\text { metered } \\
\text { dose inhaler } \\
\text { (Co-Suspension }{ }^{\mathrm{TM}} \\
\text { Delivery } \\
\text { Technology) }\end{array}$ \\
\hline $\begin{array}{l}\text { Hanania et al } \\
(2017)^{57}\end{array}$ & NCT0I970878 & $\begin{array}{l}\text { Multicenter, randomized, } \\
\text { double-blind, parallel- } \\
\text { group, active-controlled }\end{array}$ & 28 & 2,816 & $\begin{array}{l}\text { Glycopyrronium } \% \\
\text { formoterol } \\
\text { |4.4/9.6 } \mu \mathrm{g}\end{array}$ & $\begin{array}{l}\text { Pressurised } \\
\text { metered } \\
\text { dose inhaler } \\
\text { (Co-Suspension } \\
\text { Delivery } \\
\text { Technology) }\end{array}$ \\
\hline
\end{tabular}

Notes: "In German sites only, $\mathrm{FEV}_{1} \geq 30 \%$. ${ }^{\circ}$ Equivalent to glycopyrrolate $18 \mu \mathrm{g}$.

Abbreviations: $\mathrm{FEV}_{1}$, forced expiratory volume in I second; FVC, forced vital capacity; NA, not available. 


\begin{tabular}{|c|c|c|c|c|c|c|c|}
\hline $\begin{array}{l}\text { Administration } \\
\text { regimen }\end{array}$ & Patients characteristics & $\begin{array}{l}\text { Age } \\
\text { (years) }\end{array}$ & $\begin{array}{l}\text { Male } \\
(\%)\end{array}$ & $\begin{array}{l}\text { Current } \\
\text { smokers } \\
(\%)\end{array}$ & $\begin{array}{l}\text { Smoking } \\
\text { history } \\
\text { (pack- } \\
\text { years) }\end{array}$ & $\begin{array}{l}\text { Post } \\
\text { bronchodilator } \\
\text { FEV } \\
\text { (\% predicted) }\end{array}$ & $\begin{array}{l}\text { Jadad } \\
\text { score }\end{array}$ \\
\hline Once daily & $\begin{array}{l}\text { Moderate-to-very severe } \\
\text { COPD (post-bronchodilator } \\
\text { FEV,/FVC }<0.7 ; \mathrm{FEV}_{1}<80 \% \\
\text { predicted) }\end{array}$ & 69.8 & 95.2 & 28.1 & 60.3 & 59.4 & 4 \\
\hline Once daily & $\begin{array}{l}\text { COPD (post-bronchodilator } \\
\mathrm{FEV}_{\mathrm{l}} / \mathrm{FVC}<0.7 ; \mathrm{FEV}_{1} \geq 30 \% \\
\text { and }<80 \% \text { predicted) }\end{array}$ & 64.8 & 68.3 & NA & $>10$ & NA & 4 \\
\hline Once daily & $\begin{array}{l}\text { COPD (post-bronchodilator } \\
\mathrm{FEV}_{\mathrm{I}} / \mathrm{FVC}<0.7 ; \mathrm{FEV}_{1}<80 \% \\
\text { predicted) }^{\#}\end{array}$ & 61.1 & 58.9 & 62.6 & NA & 54.0 & 3 \\
\hline Once daily & $\begin{array}{l}\text { Moderate-to-very severe } \\
\text { COPD (post-bronchodilator } \\
\mathrm{FEV}_{\mathrm{l}} / \mathrm{FVC}<0.7 \text {; and } \\
\mathrm{FEV}_{1}<80 \% \text { predicted) }\end{array}$ & 64.0 & 72.9 & 37.0 & $>10$ & 50.0 & 3 \\
\hline Once daily & $\begin{array}{l}\text { Moderate-to-severe COPD } \\
\text { (post-bronchodilator FEV } / \\
\text { FVC }<0.7 ; \mathrm{FEV}_{1} \geq 30 \% \\
\text { and }<80 \% \text { predicted) }\end{array}$ & 64.8 & 61.2 & 47.7 & $>10$ & 55.1 & 3 \\
\hline Once daily & $\begin{array}{l}\text { COPD (post-bronchodilator } \\
\mathrm{FEV}_{1} / \mathrm{FVC}<0.7 ; \mathrm{FEV}_{1} \leq 70 \% \\
\text { predicted) }\end{array}$ & 63.3 & 70.7 & 48.2 & 46.0 & 47.6 & 4 \\
\hline Once daily & COPD (categories B or D) & 63.7 & 67.4 & 44.3 & 45.4 & 47.3 & 5 \\
\hline Once daily & $\begin{array}{l}\text { COPD (pre- and post- } \\
\text { albuterol [salbutamol] } \mathrm{FEV}_{1} / \\
\mathrm{FVC}<0.7 \text {; post-albuterol } \\
\mathrm{FEV}_{1} \leq 70 \% \text { predicted) }\end{array}$ & 63.4 & 59 & 53.5 & 38.6 & 47.5 & 4 \\
\hline Once daily & $\begin{array}{l}\text { Moderate-to-severe COPD } \\
\text { (post-bronchodilator FEV } / \\
\text { FVC }<0.7 ; \mathrm{FEV}_{1} \leq 70 \% \\
\text { predicted) }\end{array}$ & 63.2 & 70 & 55 & 41.3 & 47.8 & 3 \\
\hline Once daily & $\begin{array}{l}\text { COPD (postalbuterol FEV / } \\
\text { FVC }<0.7 \text {; postalbuterol } \\
\mathrm{FEV}_{1} \leq 70 \% \text { predicted; } \\
\text { dyspnea score } \geq 2 \text { ) }\end{array}$ & 64.2 & 93 & 31.5 & 37.4 & NA & 4 \\
\hline Once daily & $\begin{array}{l}\text { Moderate-to-severe stable } \\
\text { COPD (post-bronchodilator } \\
\mathrm{FEV}_{1} / \mathrm{FVC}<0.7 ; \mathrm{FEV}_{1}>35 \% \\
\text { and }<70 \% \text { predicted) }\end{array}$ & 62.0 & 56.4 & 62.0 & 48.1 & 51.3 & 4 \\
\hline Twice daily & $\begin{array}{l}\text { Moderate-to-very severe } \\
\text { COPD (post-bronchodilator } \\
\mathrm{FEV}_{\mathrm{l}} / \mathrm{FVC}<0.7 ;<80 \% \\
\text { predicted and } \geq 750 \mathrm{~mL} \\
\text { if } \mathrm{FEV}_{1}<30 \% \text { of predicted } \\
\text { normal value) }\end{array}$ & 62.9 & 55.4 & 53.8 & 51.2 & 51.5 & 4 \\
\hline Twice daily & $\begin{array}{l}\text { Moderate-to-very severe } \\
\text { COPD (post-bronchodilator } \\
\mathrm{FEV}_{\mathrm{l}} / \mathrm{FVC}<0.7 ;<80 \% \\
\text { predicted and } \geq 750 \mathrm{~mL} \\
\text { if } \mathrm{FEV},<30 \% \text { of predicted } \\
\text { normal value) }\end{array}$ & 62.8 & 55.3 & 54.0 & 51.0 & 43.1 & 4 \\
\hline
\end{tabular}


identifying the loops in the treatment network where evidence was inconsistent. ${ }^{34}$ Results of the network meta-analysis are expressed as relative effect and 95\% credible level. The probability that each intervention arm was the most effective was calculated by counting the proportion of iterations of the chain in which each intervention arm had the highest mean difference, and the surface under the cumulative ranking curve (SUCRA), representing the summary of these probabilities, was also calculated. The SUCRA is $100 \%$ when a treatment is certain to be the best, and $0 \%$ when a treatment is certain to be the worst. ${ }^{29}$

OpenMetaAnalyst (open-source, software for advanced pair-wise meta-analysis, available at http://www.cebm. brown.edu/openmeta/index.html) and GeMTC (open-source, software for advanced network meta-analysis, available at https://gemtc.drugis.org) were used for performing the meta-analysis, GraphPad Prism (GraphPad Software, La Jolla, CA, US) software to graph the data, and GRADEpro GDT (GRADEpro Guideline Development Tool. McMaster University, Hamilton, Canada, 2015, developed by Evidence Prime, Inc. Available at gradepro.org) to assess the quality of evidence. ${ }^{21,32,35}$ The statistical significance was assessed for $P<0.05$, and moderate-to-high levels of heterogeneity were considered for $I^{2}>50 \%$.

\section{Results}

\section{Studies characteristics}

Results obtained from 26,650 COPD patients (19,157 treated with an FDC, 7,197 treated with an LABA, 5,990 treated with an LAMA, and 4,306 treated with placebo) were selected from 23 studies, including 29 RCTs: four on A/F 400/12 FDC, ${ }^{36-39}$ two on G/I 15.6/27.5 FDC, ${ }^{40}$ four on G/I 50/110 FDC, ${ }^{41-44}$ nine on T/O 5/5 FDC, ${ }^{45-50}$ seven on U/V 62.5/25 FDC, ${ }^{6,51-55}$ and three on G/F 14.4/9.6. ${ }^{56,57}$ All RCTs were randomized and blinded, were published between 2013 and 2017, the period of treatment ranged from 2 to 64 weeks, and they were characterized by a Jadad score $\geq 3$. Overall, the inclusion criteria and population characteristics of the analyzed studies were homogeneous. More details on studies characteristics are reported in Table 1.

\section{Pair-wise meta-analysis} Impact of LABA/LAMA FDCs on cardiovascular SAEs

Raw data concerning the cardiovascular disorders that occurred during the RCTs have been extracted from the SAEs files of the ClinicalTrials.gov database. All studies reported suitable data on cardiovascular safety.
The overall pair-wise meta-analysis indicated that LABA/LAMA FDCs did not significantly $(P>0.05)$ modulate the risk of cardiovascular SAEs in COPD patients, compared with respective monocomponents (FDCs versus monocomponents: RR 0.94, 95\% CI 0.79-1.13, I $2 \%$; FDCs versus LABAs: RR 0.98, 95\% CI 0.72-1.34, I2 9\%; FDCs versus LAMAs: RR 0.90, 95\% CI 0.71-1.13, $I^{2} 0 \%$ ) (Figure 2A). The subset analysis on specific FDCs showed a signal $(P=0.077)$ of protection against cardiovascular SAEs for A/F 400/12 versus monocomponents (RR 0.61, 95\% CI 0.35-1.06, $\left.I^{2} 0 \%\right)$, whereas a significant $(P<0.05)$ risk was detected for $\mathrm{G} / \mathrm{F}$ 14.4/9.6 versus monocomponents (RR 1.36, 95\% CI 1.00-1.85, $I^{2} 0 \%$ ). G/I 15.6/27.5, G/I 50/110 FDC, U/V 62.5/25, and T/O 5/5 FDCs had no significant $(P>0.05)$ impact on cardiovascular safety (Figure 2B).

In any case, the pooled analysis indicated that $34.88 \%$ of cardiovascular SAEs occurred with rare frequency $(\geq 1 / 10,000$ to $<1 / 1,000)$ and $14.20 \%$ with uncommon frequency $(\geq 1 / 1,000$ to $<1 / 100)$, whereas for $50.93 \%$ of cardiovascular SAEs, the frequency could not be estimated from the available data.

The three most frequent cardiovascular SAEs were atrial fibrillation (overall: 0.39\%), myocardial infarction (overall: $0.27 \%$ ), and coronary artery disease (overall: $0.26 \%$ ), with no difference among LABA/LAMA FDCs, monocomponents and placebo. Details on the frequency of specific cardiovascular SAEs are reported in Table 2.

\section{Impact of LABA/LAMA FDCs on mortality}

LABA/LAMA FDCs did not significantly $(P>0.05)$ influence the risk of death in COPD patients, compared with respective monocomponents (FDCs versus monocomponents: RR 0.89 , 95\% CI 0.51-1.56, I² 0\%; FDCs versus LABAs: RR 0.87, 95\% CI 0.38-1.98, $I^{2}$ 9\%; FDCs versus LAMAs: RR $0.91,95 \%$ CI $\left.0.43-1.95, I^{2} 0 \%\right)$. No impact on the risk of death was detected for each specific FDC $(P>0.05$ versus monocomponents).

\section{Network meta-analysis}

The ranking plot resulting from the network meta-analysis identified three distinct clusters of safety with regard to the risk of cardiovascular SAEs. Specifically, A/F 400/12 and T/O 5/5 were the safest FDCs, followed by U/V 62.5/25 which showed a safety profile similar to that of placebo, whereas G/F 14.4/9.6, G/I 15.6/27.5, and G/I 50/110 were the least safe FDCs (Figure 3 ). The SUCRA values of the cardiovascular safety profile are reported in Table 3. 


\section{Bias and quality of evidence}

No heterogeneity was detected in the pair-wise meta-analysis, and the consistency/inconsistency analysis of the network meta-analysis indicated that all the points fitted adequately with the line of equality ( $R^{2} 0.99$; slope 1.02$)$.

Overall, this meta-analysis met a reasonable OIS to ensure a very good (probability of observing $30 \%$ overestimation for $\tau^{2}=0.25:<1 \%$ at true relative risk reduction $10 \%$ ) to excellent (probability of observing 20\% overestimation for $\tau^{2}=0.05:<1 \%$ at true relative risk reduction $0 \%$ ) low risk of observing an overestimated intervention effect due to random errors.

The analysis of bias carried out via the visual inspection of the funnel plot evidenced neither dispersion nor asymmetry
(Figure 4A), whereas Egger's tests indicated that smaller studies might have weakly, although significantly (asymmetry coefficient: $0.071 \pm 0.068 ; P<0.1$ ), distorted the results of this meta-analysis by inducing a greater effect estimate (Figure 4B). The GRADE approach indicated high quality of evidence $(\oplus \oplus \oplus \oplus)$ for the safety profile of LABA/LAMA FDCs in COPD patients resulting from this meta-analysis.

\section{Discussion}

The findings of this quantitative synthesis indicate that the LABA/LAMA FDC is a safe therapeutic approach in COPD patients. The safety profile resulting from the pair-wise meta-analysis shows that combining an LABA with an

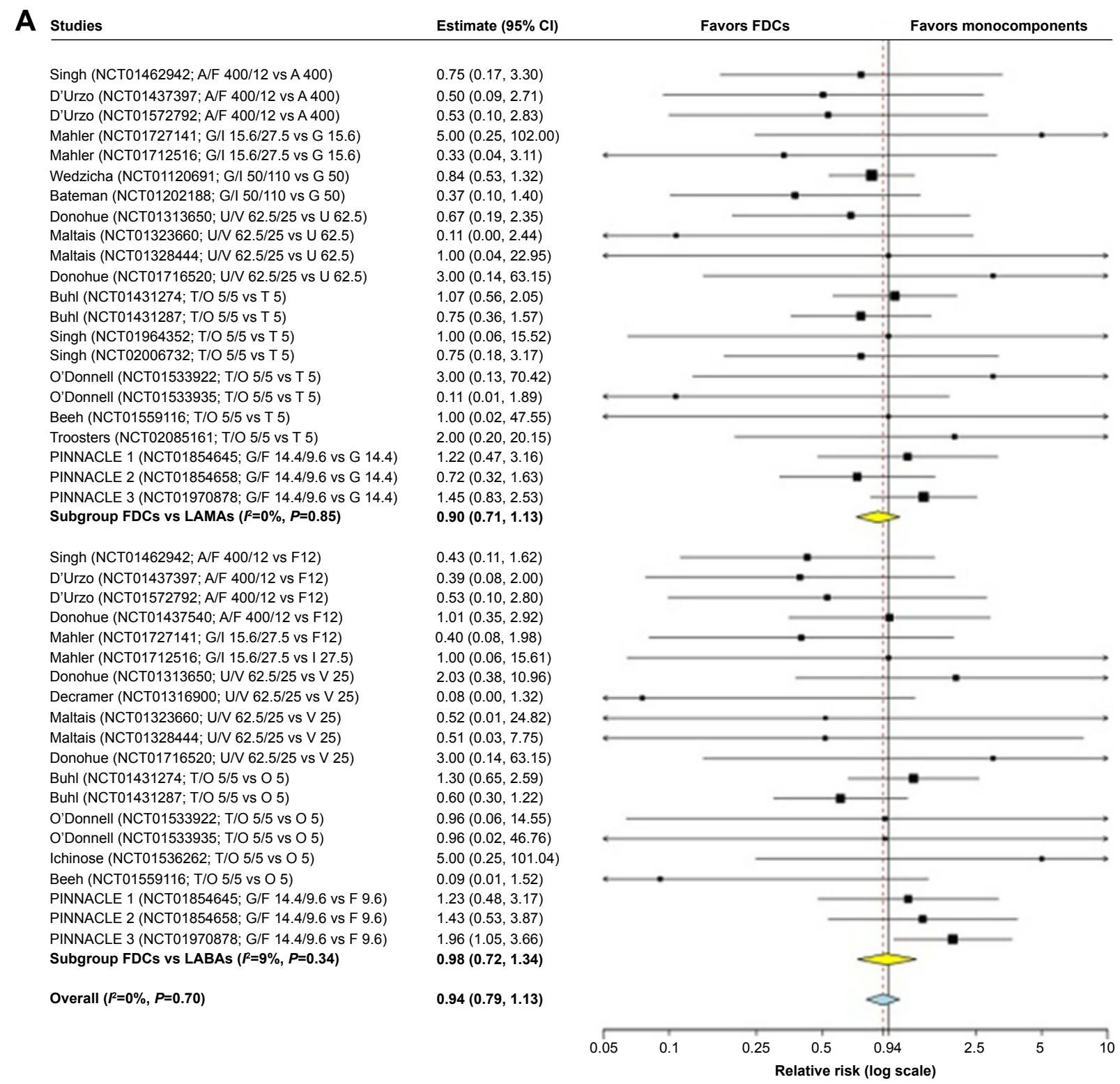

Figure 2 (Continued) 
B

Studies

Singh (NCT01462942; A/F 400/12 vs A 400)

Singh (NCT01462942; A/F 400/12 vs F 12)

D'Urzo (NCT01437397; A/F 400/12 vs A 400)

D'Urzo (NCT01437397; A/F 400/12 vs F 12)

D'Urzo (NCT01572792; A/F 400/12 vs A 400)

D'Urzo (NCT01572792; A/F 400/12 vs F 12)

Donohue (NCT01437540; A/F 400/12 vs F 12)

Subgroup $A / F 400 / 12$ vs monocomponents $(R=0 \%, P=0.95)$

Mahler (NCT01727141; G/l 15.6/27.5 vs G 15.6)

Mahler (NCT01727141; G/l 15.6/27.5 vs I 27.5)

Mahler (NCT01712516; G/l 15.6/27.5 vs G 15.6)

Mahler (NCT01712516; G/I 15.6/27.5 vs I 27.5)

Subgroup $\mathrm{G} / \mathrm{I} 15.6 / 27.5$ vs monocomponents $(R=0 \%, P=0.47)$

Wedzicha (NCT01120691; G/l 50/110 vs G 50)

Bateman (NCT01202188; G/I 50/110 vs G 50)

Subgroup $\mathrm{G} / \mathrm{l} 5 / 110$ vs monocomponents $(R=22 \%, P=0.26)$

Donohue (NCT01313650: U/V 62.5/25 vs U 62.5)

Donohue (NCT01313650; U/V 62.5/25 vs V 25)

Decramer (NCT01316900; U/V 62.5/25 vs V 25)

Maltais (NCT01323660; U/V 62.5/25 vs U 62.5)

Maltais (NCT01323660; U/V 62.5/25 vs V 25)

Maltais (NCT01328444; U/V 62.5/25 vs U 62.5)

Maltais (NCT01328444; U/V 62.5/25 vs $\vee 25$ )

Donohue (NCT01716520; U/V 62.5/25 vs U 62.5)

Donohue (NCT01716520; U/V 62.5/25 vs V 25)

Subgroup U/V 62.5/25 vs monocomponents $(R=0 \%, P=0.53)$

Buhl (NCT01431274; T/O 5/5 vs T 5)

Buhl (NCT01431274; T/O 5/5 vs O 5 )

Buhl (NCT01431287; T/O 5/5 vs T 5)

Buhl (NCT01431287; T/O 5/5 vs O 5 )

Singh (NCT01964352; T/O 5/5 vs T 5)

Singh (NCT02006732; T/O 5/5 vs T 5)

O'Donnell (NCT01533922; T/O 5/5 vs T 5)

O'Donnell (NCT01533922; T/O 5/5 vs O 5)

O'Donnell (NCT01533935; T/O 5/5 vs T 5)

O'Donnell (NCT01533935; T/O 5/5 vs O 5)

Ichinose (NCT01536262; T/O 5/5 vs O 5)

Beeh (NCT01559116; T/O 5/5 vs T 5)

Beeh (NCT01559116; T/O 5/5 vs O 5)

Troosters (NCT02085161; T/O 5/5 vs T 5 )

Subgroup $\mathrm{T} / \mathrm{O} 5 / 5$ vs monocomponents $(R=0 \%, P=0.71)$

PINNACLE 1 (NCT01854645; G/F 14.4/9.6 vs G 14.4)

PINNACLE 1 (NCT01854645; G/F 14.4/9.6 vs F 9.6)

PINNACLE 2 (NCT01854658; G/F 14.4/9.6 vs G 14.4)

PINNACLE 2 (NCT01854658; G/F 14.4/9.6 vs F 9.6)

PINNACLE 3 (NCT01970678; G/F 14.4/9.6 vs G 14.4)

PINNACLE 3 (NCT01970678; G/F 14.4/9.6 vs F 9.6)

Subgroup $G / F$ 14.4/9.6 vs monocomponents $(R=0 \%, P=0.58)$

Overall $(P=0 \%, P=0.70)$
Estimate $(95 \% \mathrm{Cl})$

$0.75(0.17,3.30)$

$0.43(0.11,1.62)$

$0.50(0.09,2.71)$

$0.39(0.08,2.00)$

$0.53(0.10,2.83)$

$0.53(0.10,2.80)$

$1.01(0.35,2.92)$

$0.61(0.35,1.06)$

$5.00(0.25,102.00)$

$0.40(0.08,1.98)$

$0.33(0.04,3.11)$

$1.00(0.06,15.61)$

$0.62(0.21,1.85)$

$0.84(0.53,1.32)$

$0.37(0.10,1.40)$

$0.72(0.39,1.34)$

$0.67(0.19,2.35)$

$2.03(0.38,10.96)$

$0.08(0.00,1.32)$

$0.11(0.00,2.44)$

$0.52(0.01,24.82)$

$1.00(0.04,22.95)$

$0.51(0.03,7.75)$

$3.00(0.14,63.15)$

$3.00(0.14,63.15)$

$0.77(0.36,1.65)$

$1.07(0.56,2.05)$

$1.30(0.65,2.59)$

$0.75(0.36,1.57)$

$0.60(0.30,1.22)$

$1.00(0.06,15.52)$

$0.75(0.18,3.17)$

$3.00(0.13,70.42)$

$0.96(0.06,14.55)$

$0.11(0.01,1.89)$

$0.96(0.02,46.76)$

$5.00(0.25,101.04)$

$1.00(0.02,47.55)$

$0.09(0.01,1.52)$

$2.00(0.20,20.15)$

$0.89(0.65,1.23)$

$1.22(0.47,3.16)$

$1.23(0.48,3.17)$

$0.72(0.32,1.63)$

$1.43(0.53,3.87)$

$1.45(0.83,2.53)$

$1.96(1.05,3.66)$

$1.36(1.00,1.85)$

$0.94(0.79,1.13)$

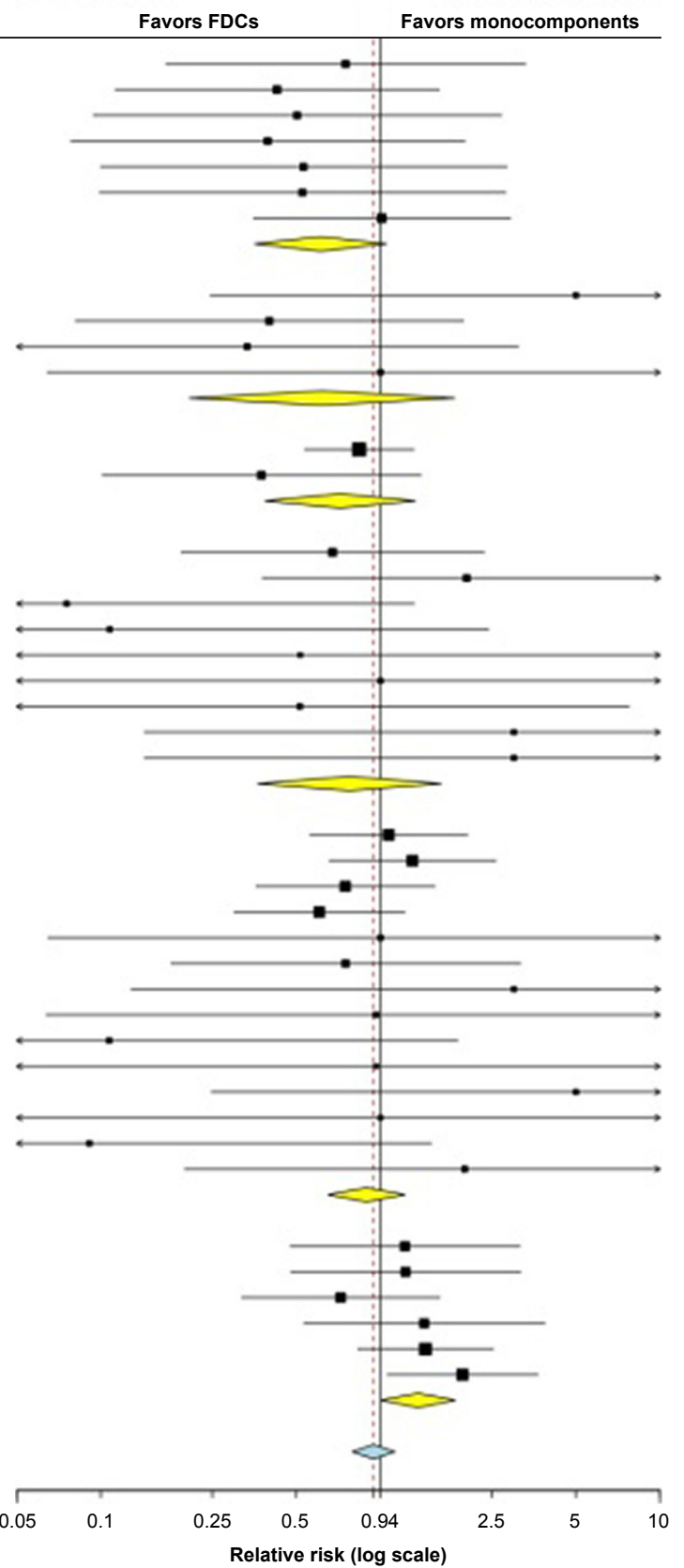

Figure 2 Forest plot of pair-wise meta-analysis of the impact of the LABA/LAMA FDCs on cardiovascular SAEs in COPD patients.

Note: Overall analysis performed by comparing LABA/LAMA FDCs versus LABAs or LAMAs (A), and subset analysis considering each specific FDC versus monocomponents (B).

Abbreviations: A, aclidinium; COPD, chronic obstructive pulmonary disease; F, formoterol; FDCs, fixed-dose combinations; G, glycopyrronium; I, indacaterol; LABAs, long-acting $\beta_{2}$-agonists; LAMAs, long-acting muscarinic antagonists; $\mathrm{O}$, olodaterol; SAEs, serious adverse events; T, tiotropium; $U$, umeclidinium; $V$, vilanterol.

LAMA does not amplify the potential cardiovascular SAEs that characterized the LABAs and LAMAs when administered as monocomponents. This result is confirmed by the subgroup analysis performed on the specific LABA/LAMA FDCs, namely G/I 15.6/27.5, G/I 50/110, U/V 62.5/25 and $\mathrm{T} / \mathrm{O} 5 / 5$, but not for $\mathrm{A} / \mathrm{F} 400 / 12$ and $\mathrm{G} / \mathrm{F} 14.4 / 9.6$. In particular, A/F 400/12 provided a strong signal of protection against cardiovascular SAEs compared with monocomponents, whereas a higher risk of cardiovascular SAEs was detected for $\mathrm{G} / \mathrm{F}$ 14.4/9.6.

Therefore, in order to better characterize the safety profile of the LABA/LAMA FDCs at the currently approved doses, we also performed a network meta-analysis using the placebo arm as the common intervention among the RCTs included 
Table 2 Pooled analysis of cardiovascular SAEs extracted from the ClinicalTrials.gov repository database and grouped by frequency in agreement with the EMA guideline ${ }^{81}$

\begin{tabular}{|c|c|c|c|c|c|c|c|c|c|}
\hline $\begin{array}{l}\text { Cardiovascular } \\
\text { SAEs }\end{array}$ & $\begin{array}{l}\text { LABA/LAMA } \\
\text { FDCs }\end{array}$ & LABAs & LAMAs & Placebo & $\begin{array}{l}\text { Cardiovascular } \\
\text { SAEs }\end{array}$ & $\begin{array}{l}\text { LABA/LAMA } \\
\text { FDCs }\end{array}$ & LABAs & LAMAs & Placebo \\
\hline $\begin{array}{l}\text { Acute coronary } \\
\text { syndrome }\end{array}$ & + & + & + & ND & Extrasystoles & + & ND & ND & ND \\
\hline $\begin{array}{l}\text { Acute myocardial } \\
\text { infarction }\end{array}$ & ++ & ++ & ++ & ++ & $\begin{array}{l}\text { Femoral artery } \\
\text { occlusion }\end{array}$ & + & ND & ND & ND \\
\hline Angina pectoris & ++ & ++ & ++ & ++ & Hypertension & + & ++ & ++ & ++ \\
\hline Angina unstable & + & + & + & ++ & Hypertensive crisis & ND & + & + & ND \\
\hline Aortic aneurysm & + & ++ & + & ND & $\begin{array}{l}\text { Hypertensive } \\
\text { emergency }\end{array}$ & + & ND & ND & ND \\
\hline $\begin{array}{l}\text { Aortic aneurysm } \\
\text { rupture }\end{array}$ & + & ND & ND & ND & Hypotension & + & ++ & ND & + \\
\hline Aortic dissection & ND & ND & + & ND & Iliac artery stenosis & + & ND & ND & ND \\
\hline Aortic stenosis & ND & + & + & + & $\begin{array}{l}\text { Intermittent } \\
\text { claudication }\end{array}$ & ND & + & + & ND \\
\hline Arrhythmia & ND & ND & ND & + & $\begin{array}{l}\text { Ischemic } \\
\text { cardiomyopathy }\end{array}$ & + & ND & + & + \\
\hline Arterial disorder & ND & + & ND & ND & $\begin{array}{l}\text { Left ventricular } \\
\text { dysfunction }\end{array}$ & + & ND & ND & + \\
\hline $\begin{array}{l}\text { Arterial occlusive } \\
\text { disease }\end{array}$ & + & + & ND & ND & Leriche syndrome & ND & + & ND & ND \\
\hline Arterial stenosis & ND & ND & + & ND & Malignant hypertension & + & ND & ND & ND \\
\hline Arteriosclerosis & + & ND & ND & ND & Mitral valve stenosis & ND & + & ND & ND \\
\hline $\begin{array}{l}\text { Arteriosclerosis } \\
\text { coronary artery }\end{array}$ & + & + & + & + & Myocardial infarction & ++ & + & ++ & ++ \\
\hline Atrial fibrillation & ++ & ++ & ++ & ++ & Myocardial ischemia & ++ & ND & + & ++ \\
\hline Atrial flutter & + & ND & ++ & ND & Palpitations & ND & ND & + & ND \\
\hline Atrial tachycardia & + & ND & ND & ND & Pericardial effusion & ND & ND & + & ND \\
\hline Atrioventricular block & ND & + & ND & ND & Pericarditis & ND & ND & + & ND \\
\hline $\begin{array}{l}\text { Atrioventricular block } \\
\text { complete }\end{array}$ & ND & ND & + & + & $\begin{array}{l}\text { Peripheral arterial } \\
\text { occlusive disease }\end{array}$ & + & + & + & ND \\
\hline $\begin{array}{l}\text { Atrioventricular block } \\
\text { second degree }\end{array}$ & ND & + & + & ND & $\begin{array}{l}\text { Peripheral artery } \\
\text { aneurysm }\end{array}$ & ND & + & + & ND \\
\hline Bradycardia & + & ND & ++ & + & $\begin{array}{l}\text { Peripheral artery } \\
\text { stenosis }\end{array}$ & + & ND & ND & ND \\
\hline $\begin{array}{l}\text { Bundle branch block } \\
\text { left }\end{array}$ & ND & + & ND & ND & $\begin{array}{l}\text { Peripheral artery } \\
\text { thrombosis }\end{array}$ & ND & + & ND & ND \\
\hline Cardiac arrest & ++ & ++ & ++ & ++ & Peripheral ischemia & + & + & ND & + \\
\hline Cardiac disorder & + & ND & ND & ND & $\begin{array}{l}\text { Peripheral vascular } \\
\text { disorder }\end{array}$ & ++ & ++ & ND & ND \\
\hline Cardiac failure & + & ++ & ++ & ++ & Phlebitis deep & + & ND & ND & ND \\
\hline Cardiac failure acute & + & + & + & ND & Right ventricular failure & ND & ND & + & + \\
\hline $\begin{array}{l}\text { Cardiac failure } \\
\text { chronic }\end{array}$ & + & ND & ND & ND & Shock & ND & + & ND & ND \\
\hline $\begin{array}{l}\text { Cardiac failure } \\
\text { congestive }\end{array}$ & ++ & + & ++ & ND & Shock hemorrhagic & + & ND & ND & ND \\
\hline $\begin{array}{l}\text { Cardio-respiratory } \\
\text { arrest }\end{array}$ & ++ & + & ++ & ND & Sick sinus syndrome & ND & ND & + & + \\
\hline Cardiogenic shock & + & ND & ND & ND & Sinus tachycardia & + & ND & ND & ND \\
\hline Cardiomyopathy & ND & ND & + & + & $\begin{array}{l}\text { Supraventricular } \\
\text { extrasystoles }\end{array}$ & ND & + & ND & ND \\
\hline $\begin{array}{l}\text { Cardiopulmonary } \\
\text { failure }\end{array}$ & + & ND & + & ND & $\begin{array}{l}\text { Supraventricular } \\
\text { tachycardia }\end{array}$ & + & + & + & + \\
\hline Circulatory collapse & ND & ND & + & ND & Tachycardia & ND & ND & + & ND \\
\hline Cor pulmonale & ND & ND & + & ND & Thrombophlebitis & ND & + & ND & ND \\
\hline
\end{tabular}


Table 2 (Continued)

\begin{tabular}{|c|c|c|c|c|c|c|c|c|c|}
\hline $\begin{array}{l}\text { Cardiovascular } \\
\text { SAEs }\end{array}$ & $\begin{array}{l}\text { LABA/LAMA } \\
\text { FDCs }\end{array}$ & LABAs & LAMAs & Placebo & $\begin{array}{l}\text { Cardiovascular } \\
\text { SAEs }\end{array}$ & $\begin{array}{l}\text { LABA/LAMA } \\
\text { FDCs }\end{array}$ & LABAs & LAMAs & Placebo \\
\hline $\begin{array}{l}\text { Cor pulmonale } \\
\text { chronic }\end{array}$ & + & ND & ND & ND & $\begin{array}{l}\text { Thrombophlebitis } \\
\text { superficial }\end{array}$ & + & ND & ND & ND \\
\hline $\begin{array}{l}\text { Coronary artery } \\
\text { disease }\end{array}$ & ++ & ++ & ++ & ++ & Thrombosis & + & ND & ND & ND \\
\hline $\begin{array}{l}\text { Coronary artery } \\
\text { occlusion }\end{array}$ & + & ND & + & ND & Torsade de pointes & ND & + & ND & ND \\
\hline $\begin{array}{l}\text { Coronary artery } \\
\text { stenosis }\end{array}$ & + & ND & ND & ND & $\begin{array}{l}\text { Ventricular } \\
\text { extrasystoles }\end{array}$ & ND & ND & ND & + \\
\hline Death & ++ & ++ & ++ & ++ & Ventricular fibrillation & ND & + & ND & ND \\
\hline Deep vein thrombosis & ++ & + & + & + & Ventricular tachycardia & + & + & ND & ND \\
\hline Essential hypertension & + & ND & + & ND & & & & & \\
\hline
\end{tabular}

Notes: ++: uncommon $(\geq \mathrm{I} / \mathrm{I}, 000$ to $<\mathrm{I} / 100) ;+$ : rare $(\geq \mathrm{I} / 10,000$ to $<\mathrm{I} / \mathrm{I}, 000)$.

Abbreviations: EMA, European Medicine Agency; FDCs, fixed-dose combinations; LABA, long-acting $\beta_{2}$-agonist; LAMA, long-acting muscarinic antagonist; ND, not detectable (frequency not known); SAEs, serious adverse events.

in this study. Such a network approach allowed us to perform an indirect comparison of the investigated interventions that were not previously studied in a head-to-head fashion. ${ }^{12}$ Results of the network meta-analysis generally confirm those of the pair-wise meta-analysis. The risk of cardiovascular SAEs was lower in COPD patients treated with A/F 400/12 and T/O 5/5 FDCs than in the placebo arm, it was similar to placebo for U/V 62.5/25 FDCs, and higher than placebo for G/F 14.4/9.6, G/I 15.6/27.5, G/I 50/110 FDCs.

Indeed, the network meta-analysis would provide more refined estimates if data on direct comparison between

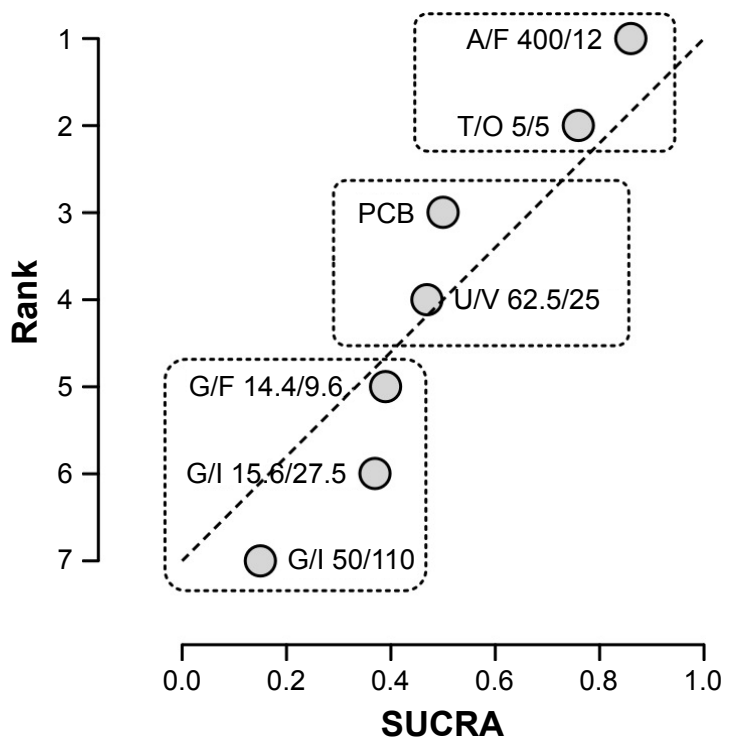

Figure 3 Ranking plot of the network on the cardiovascular safety profile of LABA/ LAMA FDCs versus placebo in COPD patients.

Note: Treatments have been plotted on the $X$-axis according to SUCRA (score of I being the safest) and on the $\mathrm{Y}$-axis according to the rank of being the best treatment (score of I being the safest).

Abbreviations: $A$, aclidinium; COPD, chronic obstructive pulmonary disease; $F$, formoterol; FDC, fixed-dose combination; G, glycopyrronium; I, indacaterol; LABA, long-acting $\beta_{2}$-agonist; LAMA, long-acting muscarinic antagonists; $O$, olodaterol; PCB, placebo; SUCRA, surface under the cumulative ranking curve; $T$, tiotropium; $\mathrm{U}$, umeclidinium; $\mathrm{V}$, vilanterol.
LABA/LAMA FDCs were available but, unfortunately, results on head-to-head RCTs are not currently available. However, studies comparing U/V with $\mathrm{T} / \mathrm{O}$ and $\mathrm{G} / \mathrm{F}$ with U/V are ongoing (ClinicalTrials.gov identifiers: NCT02799784 and NCT03162055, respectively), and two further RCTs aiming to compare G/I with U/V have been completed but study results have not yet been posted (ClinicalTrials.gov identifiers: NCT02487498 and NCT02487446). When these results are accessible to independent researchers, closed loops might be created into the network to further improve the consistency of the indirect comparisons. ${ }^{58}$

In any case, we have to recognize that, although a rank of safety profile exists among the LABA/LAMA FDCs, no difference on the risk of death was detected for each specific FDC. Furthermore, mortality was detected with the same uncommon frequency in COPD patients receiving LABA/ LAMA FDCs as in those treated with monocomponents and placebo. Similarly, the most frequent cardiovascular SAE, atrial fibrillation, had the same frequency among the treatments, including placebo, with the highest value of $<4$ cases in 1,000 patients.

Table 3 Safety profile of LABA/LAMA FDCs according to SUCRA analysis

\begin{tabular}{ll}
\hline Treatment & SUCRA value (\%) \\
\hline A/F 400/12 & 86.00 \\
T/O 5/5 & 75.67 \\
Placebo & 49.67 \\
U/V 62.5/25 & 47.33 \\
G/F I4.4/9.6 & 39.00 \\
G/I I5.6/27.5 & 37.00 \\
G/I 50/IIO & 15.00
\end{tabular}

Abbreviations: A, aclidinium; F, formoterol; FDCs, fixed-dose combinations; G, glycopyrronium; I, indacaterol; LABA, long-acting $\beta_{2}$-agonist; LAMA, long-acting muscarinic antagonist; $O$, olodaterol; SUCRA, surface under the cumulative ranking curve; $\mathrm{T}$, tiotropium; $\mathrm{U}$, umeclidinium; $\mathrm{V}$, vilanterol. 

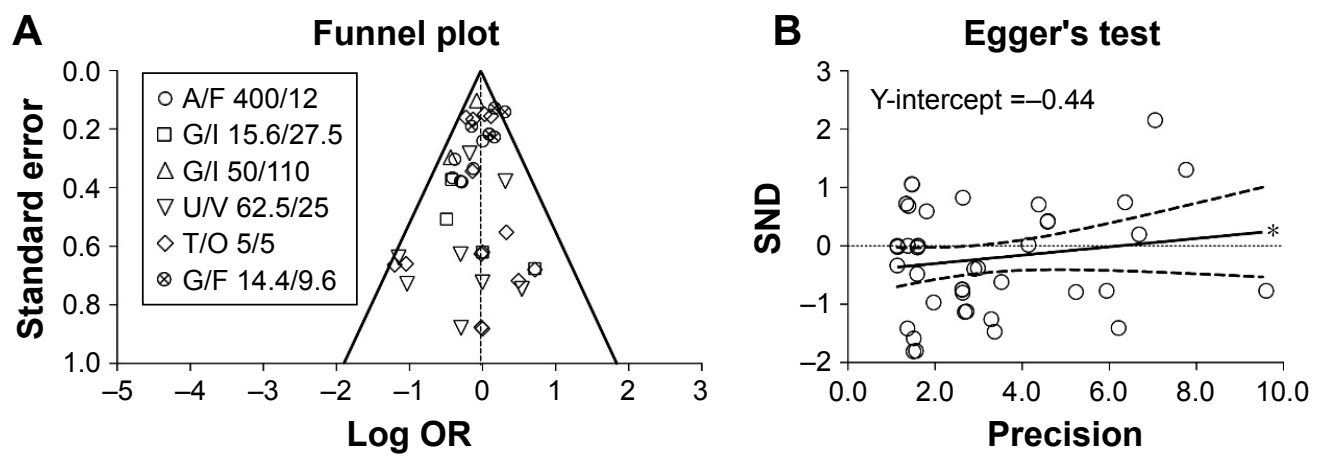

Figure 4 Publication bias assessment via funnel plot $(\mathbf{A})$ and Egger's test $(\mathbf{B})$ for the impact of LABA/LAMA FDCs on cardiovascular SAEs in COPD patients, versus respective monocomponents.

Note: $* P<0.1$.

Abbreviations: A, aclidinium; COPD, chronic obstructive pulmonary disease; F, formoterol; FDCs, fixed-dose combinations; G, glycopyrronium; I, indacaterol; LABA, long-acting $\beta_{2}$-agonist; LAMA, long-acting muscarinic antagonists; O, olodaterol; OR, odds ratio; SAEs, serious adverse events; SND, standard normal deviate; T, tiotropium; $\mathrm{U}$, umeclidinium; $\mathrm{V}$, vilanterol.

From a strictly analytical point of view, the detected rare/ uncommon frequency of cardiovascular SAEs makes it difficult to get concordant results and, since there is no perfect meta-analysis technique for low frequency events, the findings on statistical significance or signal of significance must be interpreted cautiously ${ }^{59,60} \mathrm{In}$ fact, although this quantitative synthesis provides a high quality of evidence, we have detected a certain level of bias related with the so-called "small study effect", leading to a higher risk of cardiovascular SAEs in smaller RCTs compared with that observed in larger studies. This bias, together with the fact that few studies have been performed on the G/F 14.4/9.6 FDC, may have caused an imbalance in the effect estimates in favour of $\mathrm{A} / \mathrm{F} 400 / 12$ and against G/F 14.4/9.6 FDCs.

Furthermore, we cannot exclude that the occurrence of rare/uncommon cardiovascular SAEs may be related to various features of the individual patient. Although the safety profile is an essential element for approval by regulatory authorities, pivotal RCTs include a small and highly selected fraction of the patients. Indeed, the populations selected for RCTs only partially represent the real-life population, as it has been extensively proved that in large populations of individuals with an established diagnosis of COPD fewer than $\simeq 14 \%$ of outpatients were eligible for inclusion in RCTs ${ }^{61,62}$ In particular, COPD patients with co-morbidities are usually excluded from RCTs, and this approach may lead to potential bias considering that COPD is a risk factor for several cardiovascular diseases. ${ }^{10}$ In this regard, post-marketing surveillance and observational studies represent useful tools to adequately assess the safety profile of LABA/LAMA FDCs in the real-life population of COPD patients. ${ }^{63}$

The rank of cardiovascular safety detected in this metaanalysis may also be explained by considering the dissimilarities in the pharmacodynamic and pharmacokinetic characteristics of the individual components of any LABA/ LAMA FDC. Indeed, the cardiovascular AEs are due to absorption and systemic distribution of LABAs and LAMAs after inhalation. ${ }^{64}$ The localization of $\beta_{2}$-adrenergic receptor (AR) agonist in the heart modulates the cardiac functions, although the heart expresses a lower $\beta_{2}$-AR density than do the airways. Therefore, full (ie, formoterol, indacaterol) or near-full $\beta_{2}$-AR agonists (ie, olodaterol) would have a greater cardiac impact than partial agonists (ie, vilanterol). ${ }^{65,66} \mathrm{How}-$ ever, it is well known that desensitization through dampening of the signaling cascade or down-regulation of the number of $\beta_{2}$-ARs (ie, tachyphylaxis) after chronic $\beta_{2}$-AR agonist use might often resolve the reported side effects such as tachycardia as seen on treatment initiation. ${ }^{64}$

Also, the blockade of the $\mathrm{M}_{2}$ muscarinic receptor induced by LAMAs has the potential to cause cardiovascular AEs. However, LAMAs are characterized by important differences in dissociation half-lives for muscarinic antagonists against the $\mathrm{M}_{2}$ and $\mathrm{M}_{3}$ muscarinic receptor subtypes.$^{67} \mathrm{In}$ particular, glycopyrronium and umeclidinium are characterized by a greater selectivity for the $\mathrm{M}_{3}$ muscarinic receptor versus the $\mathrm{M}_{2}$ muscarinic receptor compared with tiotropium, and dissociate from the $M_{2}$ muscarinic receptor more readily than does tiotropium. ${ }^{68,69}$ Aclidinium is rapidly hydrolyzed into derivatives that are devoid of any affinity for all muscarinic receptor subtypes. ${ }^{10,70}$

It is intriguing that in this meta-analysis, FDCs, including glycopyrronium combined with two full/nearly full $\beta_{2}$-AR agonists (fromoterol and indacaterol) exhibited a higher risk for cardiovascular SAEs than placebo. A comprehensive analysis of clinical studies and post-marketing data has already shown that atrial fibrillation events were seen more often with glycopyrronium than with placebo, although the difference was not statistically significant. ${ }^{71}$ 
The aforementioned analysis, ${ }^{71}$ and the rare/uncommon frequency of cardiovascular SAEs detected in the present meta-analysis, suggest that there may be a different cardiovascular response to muscarinic receptors blockage and $\beta_{2}$-ARs stimulation in individual patients, that is not specific to any LAMA or LABA. It has been suggested that rare polymorphisms in regulator of G-protein signaling 2, a putative regulator of the $\mathrm{M}_{3}$ muscarinic receptor, can be associated with arrhythmias. ${ }^{72}$ Furthermore, there is evidence that $\mathrm{M}_{3}$ muscarinic receptor overexpression reduces the incidence of arrhythmias and mortality in a mouse model of myocardial ischemia-reperfusion, by protecting the myocardium from ischemia. ${ }^{73}$ It is likely that changes in this overexpression on an individual basis may induce different responses to the blockade of muscarinic receptors operated by muscarinic antagonists, considering that all of the muscarinic antagonists can cause more or less cardiovascular SAEs. ${ }^{74,75}$ On the other hand, patients who are less susceptible to desensitization, due to different genetic variants of the $\beta_{2}$-AR, are more likely to be at a higher risk of cardiovascular SAEs, particularly in individuals with long-term exposure to accumulated doses of $\beta_{2}$-AR agonist. ${ }^{76,77}$ Unfortunately, the examined RCTs were not focused on genetic variations and, consequently, patients were not stratified according to genotype. Nonetheless, we do not believe that a specific LABA/LAMA FDC may expose patients to higher risks of real SAEs than do other FDCs. Rather, we believe that there may be a different cardiovascular response to any LABA/LAMA FDC in individual patients. Therefore, it will be essential to make all possible efforts to proactively identify patients at increased risk of cardiovascular SAEs when treated with LABA/LAMA FDCs.

Finally, but not less important, also the drug formulations and the characteristics of the specific devices, that can influence the systemic drug concentrations, may have an impact on the possible occurrence of cardiovascular SAEs, and lead to potential imbalance of the safety profile in favor of some LABA/LAMA FDCs rather than others. ${ }^{78,79}$

\section{Conclusions}

This quantitative synthesis provides high quality evidence that LABA/LAMA FDC therapy is characterized by an excellent cardiovascular safety profile, at least in the COPD population enrolled in RCTs. The rare/uncommon frequency of cardiovascular SAEs suggests that the rank of safety profile across the currently approved LABA/LAMA FDCs should be interpreted with caution, and results considered exploratory in nature and hypothesis-generating. ${ }^{60}$ Although the choice of a specific LAMA/LABA FDC should not be based on any difference in the safety profile, post-marketing surveillance and observational studies may help to better define the real impact of specific LABA/LAMA FDCs with regard to the cardiovascular risk.

\section{Acknowledgments}

We thank Ms Beatrice Ludovica Ritondo (Department of Systems Medicine, University of Rome Tor Vergata, Rome, Italy) for her support in extracting the data.

\section{Author contributions}

Substantial contributions to conception and design, acquisition of data, or analysis and interpretation of data: PR, MGM, $\mathrm{JO} ; \mathrm{MC}, \mathrm{LC}$; drafting the article or revising it critically for important intellectual content: PR, MGM, JO; MC, LC; final approval of the version to be published: PR, MGM, JO; MC, $\mathrm{LC}$; agreement to be accountable for all aspects of the work in ensuring that questions related to the accuracy or integrity of any part of the work are appropriately investigated and resolved: PR, MGM, JO, MC, LC.

\section{Disclosure}

PR participated as a lecturer, speaker, and advisor in scientific meetings and courses under the sponsorship of Almirall, AstraZeneca, Biofutura, Boehringer Ingelheim, Chiesi Farmaceutici, GlaxoSmithKline, Menarini Group, Mundipharma, and Novartis; her department was funded by Almirall, Boehringer Ingelheim, Novartis, and Zambon. MGM has participated as a lecturer, speaker, and advisor in scientific meetings and courses under the sponsorship of Almirall, AstraZeneca, Boehringer Ingelheim, Chiesi Farmaceutici, GlaxoSmithKline and Novartis, and has been a consultant to Chiesi Farmaceutici. JO has no conflicts of interest in this work. MC has participated as a lecturer, speaker, and advisor in scientific meetings and courses under the sponsorship of Almirall, AstraZeneca, Biofutura, Boehringer Ingelheim, Chiesi Farmaceutici, GlaxoSmithKline, Menarini Group, Lallemand, Mundipharma, Novartis, Pfizer, Verona Pharma, and Zambon, and is or has been a consultant to Chiesi Farmaceutici, Edmond Pharma Lallemand, Novartis, Verona Pharma, and Zambon; his department was funded by Almirall, Boehringer Ingelheim, Novartis, and Zambon. LC has participated as advisor in scientific meetings under the sponsorship of Boehringer Ingelheim and Novartis, received non-financial support by AstraZeneca, received a research grant partially funded by Boehringer Ingelheim, Novartis and Almirall, and is or has been a consultant to Edmond Pharma, Zambon and Verona Pharma; his departmentwas funded by Almirall, Boehringer Ingelheim, Novartis, and Zambon. 


\section{References}

1. Cazzola M, Calzetta L, Ora J, Puxeddu E, Rogliani P, Matera MG. Searching for the synergistic effect between aclidinium and formoterol: from bench to bedside. Respir Med. 2015;109(10):1305-1311.

2. Cazzola M, Calzetta L, Page CP, et al. Pharmacological characterization of the interaction between aclidinium bromide and formoterol fumarate on human isolated bronchi. Eur J Pharmacol. 2014;745:135-143.

3. Cazzola M, Calzetta L, Puxeddu E, et al. Pharmacological characterisation of the interaction between glycopyrronium bromide and indacaterol fumarate in human isolated bronchi, small airways and bronchial epithelial cells. Respir Res. 2016;17(1):70.

4. Cazzola M, Calzetta L, Segreti A, Facciolo F, Rogliani P, Matera MG. Translational study searching for synergy between glycopyrronium and indacaterol. COPD. 2015;12(2):175-181.

5. Calzetta L, Rogliani P, Mattei M, et al. Pharmacological characterization of the interaction between tiotropium and olodaterol administered at 5:5 concentration-ratio in equine bronchi. COPD. 2017;14(5): 526-532.

6. Donohue JF, Singh D, Munzu C, Kilbride S, Church A. Magnitude of umeclidinium/vilanterol lung function effect depends on monotherapy responses: Results from two randomised controlled trials. Respir Med. 2016;112:65-74

7. Calzetta L, Rogliani P, Facciolo F, Rendina E, Cazzola M, Matera MG. Pharmacological characterization of the interaction between umeclidinium and vilanterol in human bronchi. Eur J Pharmacol. 2017;812: 147-154.

8. Calzetta L, Matera MG, Cazzola M. Pharmacological interaction between LABAs and LAMAs in the airways: optimizing synergy. Eur J Pharmacol. 2015;761:168-173.

9. Cazzola M, Rogliani P, Matera MG. Escalation and de-escalation of therapy in COPD: myths, realities and perspectives. Drugs. 2015;75(14): 1575-1585.

10. Matera MG, Rogliani P, Calzetta L, Cazzola M. Safety considerations with dual bronchodilator therapy in COPD: an update. Drug Saf. 2016;39(6):501-508.

11. Calzetta L, Rogliani P, Matera MG, Cazzola M. A systematic review with meta-analysis of dual bronchodilation with LAMA/LABA for the treatment of stable COPD. Chest. 2016;149(5):1181-1196.

12. Jansen JP, Naci H. Is network meta-analysis as valid as standard pairwise meta-analysis? It all depends on the distribution of effect modifiers. BMC Med. 2013;11(1):159.

13. Moher D, Liberati A, Tetzlaff J, Altman DG, Group P. Preferred reporting items for systematic reviews and meta-analyses: the PRISMA Statement. Open Med. 2009;3(3):e123-e130.

14. Moher D, Shamseer L, Clarke M, et al. Preferred reporting items for systematic review and meta-analysis protocols (PRISMA-P) 2015 statement. Syst Rev. 2015;4(1):1.

15. Rogliani P, Calzetta L, Cavalli F, Matera MG, Cazzola M. Pirfenidone, nintedanib and N-acetylcysteine for the treatment of idiopathic pulmonary fibrosis: a systematic review and meta-analysis. Pulm Pharmacol Ther. 2016;40:95-103.

16. Kew KM, Dias S, Cates CJ. Long-acting inhaled therapy (beta-agonists, anticholinergics and steroids) for COPD: a network meta-analysis. Cochrane Database Syst Rev. 2014;3:CD010844.

17. Calzetta L, Rogliani P, Ora J, Puxeddu E, Cazzola M, Matera MG. LABA/LAMA combination in COPD: a meta-analysis on the duration of treatment. Eur Respir Rev. 2017;26(143):160043.

18. Rodrigo GJ, Price D, Anzueto A, et al. LABA/LAMA combinations versus LAMA monotherapy or LABA/ICS in COPD: a systematic review and meta-analysis. Int J Chron Obstruct Pulmon Dis. 2017;12: 907-922.

19. Higgins JPT, Green S. Cochrane Handbook for Systematic Reviews of Interventions Version 5.1.0. The Cochrane Collaboration; 2011. Available from: www.cochrane-handbook.org. Accessed June 14, 2017.

20. Rogliani P, Calzetta L, Cazzola M, Matera MG. Drug safety evaluation of roflumilast for the treatment of COPD: a meta-analysis. Expert Opin Drug Saf. 2016;15(8):1133-1146.
21. Guyatt G, Oxman AD, Akl EA, et al. GRADE guidelines: 1. IntroductionGRADE evidence profiles and summary of findings tables. J Clin Epidemiol. 2011;64(4):383-394.

22. Cazzola M, Calzetta L, Page C, et al. Influence of N-acetylcysteine on chronic bronchitis or COPD exacerbations: a meta-analysis. Eur Respir Rev. 2015;24(137):451-461.

23. Shen Y, Cai W, Lei S, Zhang Z. Effect of high/low dose N-acetylcysteine on chronic obstructive pulmonary disease: a systematic review and meta-analysis. COPD. 2014;11(3):351-358.

24. Calzetta L, Matera MG, Braido F, et al. Withdrawal of inhaled corticosteroids in COPD: a meta-analysis. Pulm Pharmacol Ther. 2017;45: $148-158$.

25. Guevara JP, Berlin JA, Wolf FM. Meta-analytic methods for pooling rates when follow-up duration varies: a case study. BMC Med Res Methodol. 2004;4(1):17.

26. DeCoster J. Meta-analysis notes; 2004. Available from: http://www. stat-help.com/meta.pdf. Accessed June 14, 2017.

27. Turner JR, Durham TA. Meta-methodology: conducting and reporting meta-analyses. J Clin Hypertens (Greenwich). 2014;16(2):91-93.

28. Calzetta L, Roncada P, di Cave D, et al. Pharmacological treatments in asthma-affected horses: a pair-wise and network meta-analysis. Equine Vet J. 2017;49(6):710-717.

29. Calzetta L, Rogliani P, Ora J, Puxeddu E, Cazzola M, Gabriella Matera M. LABA/LAMA combination in COPD: a meta-analysis on the duration of treatment. Eur Respir Rev. 2017;26(143):160043.

30. Lu G, Ades AE. Assessing evidence inconsistency in mixed treatment comparisons. J Am Statist Assoc. 2006;101(474):447-459.

31. Spiegelhalter DJ, Abrams KR, Myles JP. Bayesian Approaches to Clinical Trials and Health-Care Evaluation. Vol 13. Chichester: Wiley; 2004.

32. van Valkenhoef G, Lu G, de Brock B, Hillege H, Ades AE, Welton NJ. Automating network meta-analysis. Res Synth Methods. 2012;3(4): 285-299.

33. Valkenhoef G, Dias S, Ades AE, Welton NJ. Automated generation of node-splitting models for assessment of inconsistency in network meta-analysis. Res Synth Methods. 2016;7(1):80-93.

34. Dias S, Welton NJ, Sutton AJ, Caldwell DM, Lu G, Ades AE. Evidence synthesis for decision making 4: inconsistency in networks of evidence based on randomized controlled trials. Med Decis Making. 2013; 33(5):641-656.

35. Wallace BC, Dahabreh IJ, Trikalinos TA, Lau J, Trow P, Schmid CH. Closing the gap between methodologists and end-users: $\mathrm{R}$ as a computational back-end. J Statist Software. 2012;49(5):1-15.

36. D’Urzo A, Rennard S, Kerwin E, et al. A randomised double-blind, placebo-controlled, long-term extension study of the efficacy, safety and tolerability of fixed-dose combinations of aclidinium/formoterol or monotherapy in the treatment of chronic obstructive pulmonary disease. Respir Med. 2017;125:39-48.

37. Donohue JF, Soong W, Wu X, Shrestha P, Lei A. Long-term safety of aclidinium bromide/formoterol fumarate fixed-dose combination: results of a randomized 1-year trial in patients with COPD. Respir Med. 2016;116:41-48.

38. Singh D, Jones PW, Bateman ED, et al. Efficacy and safety of aclidinium bromide/formoterol fumarate fixed-dose combinations compared with individual components and placebo in patients with COPD (ACLIFORM-COPD): a multicentre, randomised study. BMC Pulm Med. 2014;14:178.

39. D'Urzo AD, Rennard SI, Kerwin EM, et al. Efficacy and safety of fixed-dose combinations of aclidinium bromide/formoterol fumarate: the 24-week, randomized, placebo-controlled AUGMENT COPD study. Respir Res. 2014;15(1):123.

40. Mahler DA, Kerwin E, Ayers T, et al. FLIGHT: efficacy and safety of QVA149 (indacaterol/glycopyrrolate) versus its monocomponents and placebo in patients with COPD. Am J Respir Crit Care Med. 2015; 92(9):1068-1079.

41. Watz H, Mailander C, Baier M, Kirsten A. Effects of indacaterol/ glycopyrronium (QVA149) on lung hyperinflation and physical activity in patients with moderate to severe COPD: a randomised, placebo-controlled, crossover study (The MOVE Study). BMC Pulm Med. 2016;16(1):95. 
42. Bateman ED, Ferguson GT, Barnes N, et al. Dual bronchodilation with QVA149 versus single bronchodilator therapy: the SHINE study. Eur Respir J. 2013;42(6):1484-1494.

43. Wedzicha JA, Decramer M, Ficker JH, et al. Analysis of chronic obstructive pulmonary disease exacerbations with the dual bronchodilator QVA149 compared with glycopyrronium and tiotropium (SPARK): a randomised, double-blind, parallel-group study. Lancet Respir Med. 2013;1(3):199-209.

44. Buhl R, Gessner C, Schuermann W, et al. Efficacy and safety of oncedaily QVA149 compared with the free combination of once-daily tiotropium plus twice-daily formoterol in patients with moderate-to-severe COPD (QUANTIFY): a randomised, non-inferiority study. Thorax. 2015;70(4):311-319.

45. O'Donnell DE, Casaburi R, Frith P, et al. Effects of combined tiotropium/olodaterol on inspiratory capacity and exercise endurance in COPD. Eur Respir J. 2017;49(4):1601348.

46. Ichinose M, Kato M, Takizawa A, et al. Long-term safety and efficacy of combined tiotropium and olodaterol in Japanese patients with chronic obstructive pulmonary disease. Respir Investig. 2017;55(2):121-129.

47. Troosters T, Bourbeau J, Maltais F, et al. Enhancing exercise tolerance and physical activity in COPD with combined pharmacological and non-pharmacological interventions: PHYSACTO randomised, placebocontrolled study design. BMJ Open. 2016;6(4):e010106.

48. Beeh KM, Westerman J, Kirsten AM, et al. The 24-h lung-function profile of once-daily tiotropium and olodaterol fixed-dose combination in chronic obstructive pulmonary disease. Pulm Pharmacol Ther. 2015;32:53-59.

49. Buhl R, Maltais F, Abrahams R, et al. Tiotropium and olodaterol fixeddose combination versus mono-components in COPD (GOLD 2-4). Eur Respir J. 2015;45(4):969-979.

50. Singh D, Ferguson GT, Bolitschek J, et al. Tiotropium + olodaterol shows clinically meaningful improvements in quality of life. Respir Med. 2015;109(10):1312-1319.

51. Donohue JF, Maleki-Yazdi MR, Kilbride S, Mehta R, Kalberg C, Church A. Efficacy and safety of once-daily umeclidinium/vilanterol 62.5/25 mcg in COPD. Respir Med. 2013;107(10):1538-1546.

52. Decramer M, Anzueto A, Kerwin E, et al. Efficacy and safety of umeclidinium plus vilanterol versus tiotropium, vilanterol, or umeclidinium monotherapies over 24 weeks in patients with chronic obstructive pulmonary disease: results from two multicentre, blinded, randomised controlled trials. Lancet Respir Med. 2014;2(6):472-486.

53. Siler TM, Donald AC, O’Dell D, Church A, Fahy WA. A randomized, parallel-group study to evaluate the efficacy of umeclidinium/vilanterol $62.5 / 25 \mu \mathrm{g}$ on health-related quality of life in patients with COPD. Int J Chron Obstruct Pulmon Dis. 2016;11:971-979.

54. Zheng J, Zhong N, Newlands A, Church A, Goh AH. Efficacy and safety of once-daily inhaled umeclidinium/vilanterol in Asian patients with COPD: results from a randomized, placebo-controlled study. Int J Chron Obstruct Pulmon Dis. 2015;10:1753-1767.

55. Maltais F, Singh S, Donald AC, et al. Effects of a combination of umeclidinium/vilanterol on exercise endurance in patients with chronic obstructive pulmonary disease: two randomized, double-blind clinical trials. Ther Adv Respir Dis. 2014;8(6):169-181.

56. Martinez FJ, Rabe KF, Ferguson GT, et al. Efficacy and safety of glycopyrrolate/formoterol metered dose inhaler formulated using cosuspension delivery technology in patients with COPD. Chest. 2017; 151(2):340-357.

57. Hanania NA, Tashkin DP, Kerwin EM, et al. Long-term safety and efficacy of glycopyrrolate/formoterol metered dose inhaler using novel Co-Suspension Delivery Technology in patients with chronic obstructive pulmonary disease. Respir Med. 2017;126:105-115.

58. Salanti G. Indirect and mixed-treatment comparison, network, or multiple-treatments meta-analysis: many names, many benefits, many concerns for the next generation evidence synthesis tool. Res Synth Methods. 2012;3(2):80-97.

59. Cheng J, Pullenayegum E, Marshall JK, Iorio A, Thabane L. Impact of including or excluding both-armed zero-event studies on using standard meta-analysis methods for rare event outcome: a simulation study. BMJ Open. 2016;6(8):e010983.
60. Lane PW. Meta-analysis of incidence of rare events. Stat Methods Med Res. 2013;22(2):117-132.

61. Scichilone N, Basile M, Battaglia S, Bellia V. What proportion of chronic obstructive pulmonary disease outpatients is eligible for inclusion in randomized clinical trials? Respiration. 2014;87(1):11-17.

62. Travers J, Marsh S, Caldwell B, et al. External validity of randomized controlled trials in COPD. Respir Med. 2007;101(6):1313-1320.

63. Charles C. New options for optimal bronchodilation in chronic obstructive pulmonary disease. EMJ Respir. 2014;2:58-66.

64. Cazzola M, Page CP, Calzetta L, Matera MG. Pharmacology and therapeutics of bronchodilators. Pharmacol Rev. 2012;64(3):450-504

65. Matera MG, Calzetta L, Cazzola M. $\beta$-adrenoceptor modulation in chronic obstructive pulmonary disease: present and future perspectives. Drugs. 2013;73(15):1653-1663.

66. Ricciardolo FL, Blasi F, Centanni S, Rogliani P. Therapeutic novelties of inhaled corticosteroids and bronchodilators in asthma. Pulm Pharmacol Ther. 2015;33:1-10.

67. Cazzola M, Page C, Matera MG. Long-acting muscarinic receptor antagonists for the treatment of respiratory disease. Pulm Pharmacol Ther. 2013;26(3):307-317.

68. Buhl R, Banerji D. Profile of glycopyrronium for once-daily treatment of moderate-to-severe COPD. Int J Chron Obstruct Pulmon Dis. 2012;7:729-741.

69. Segreti A, Calzetta L, Rogliani P, Cazzola M. Umeclidinium for the treatment of chronic obstructive pulmonary disease. Expert Rev Respir Med. 2014;8(6):665-671.

70. Cazzola M, Page CP, Matera MG. Aclidinium bromide for the treatment of chronic obstructive pulmonary disease. Expert Opin Pharmacother. 2013;14(9):1205-1214.

71. D’Urzo AD, Kerwin EM, Chapman KR, et al. Safety of inhaled glycopyrronium in patients with COPD: a comprehensive analysis of clinical studies and post-marketing data. Int J Chron Obstruct Pulmon Dis. 2015;10:1599-1612.

72. Zhang P, Mende U. Functional role, mechanisms of regulation, and therapeutic potential of regulator of $\mathrm{G}$ protein signaling 2 in the heart. Trends Cardiovasc Med. 2014;24(2):85-93.

73. Liu Y, Sun L, Pan Z, et al. Overexpression of M(3) muscarinic receptor is a novel strategy for preventing sudden cardiac death in transgenic mice. Mol Med. 2011;17(11-12):1179-1187.

74. Matera MG, Rogliani P, Cazzola M. Muscarinic receptor antagonists for the treatment of chronic obstructive pulmonary disease. Expert Opin Pharmacother. 2014;15(7):961-977.

75. Singh S, Loke YK, Furberg CD. Inhaled anticholinergics and risk of major adverse cardiovascular events in patients with chronic obstructive pulmonary disease: a systematic review and meta-analysis. JAMA. 2008;300(12):1439-1450

76. Muthumala A, Drenos F, Elliott PM, Humphries SE. Role of beta adrenergic receptor polymorphisms in heart failure: systematic review and meta-analysis. Eur J Heart Fail. 2008;10(1):3-13.

77. Cazzola M, Matera MG, Donner CF. Inhaled beta2-adrenoceptor agonists: cardiovascular safety in patients with obstructive lung disease. Drugs. 2005;65(12):1595-1610.

78. Rogliani P, Calzetta L, Coppola A, et al. Optimizing drug delivery in COPD: the role of inhaler devices. Respir Med. 2017;124:6-14.

79. Cazzola M, Calzetta L, Rogliani P, Matera MG. Tiotropium formulations and safety: a network meta-analysis. Ther Adv Drug Saf. 2017; 8(1):17-30.

80. Singh D, Ferguson GT, Bolitschek J, et al. Tiotropium + olodaterol shows clinically meaningful improvements in quality of life. Respir Med. 2015;109(10):1312-1319.

81. European Commission. A guideline on summary of product characteristics (SmPC); 2009. Available from: http://ec.europa.eu/health// sites/health/files/files/eudralex/vol-2/c/smpc_guideline_rev2_en.pdf. Accessed June 22, 2017. 
International Journal of COPD

\section{Publish your work in this journal}

The International Journal of COPD is an international, peer-reviewed journal of therapeutics and pharmacology focusing on concise rapid reporting of clinical studies and reviews in COPD. Special focus is given to the pathophysiological processes underlying the disease, intervention programs, patient focused education, and self management protocols.

This journal is indexed on PubMed Central, MedLine and CAS. The manuscript management system is completely online and includes a very quick and fair peer-review system, which is all easy to use. Visit http://www.dovepress.com/testimonials.php to read real quotes from published authors 